\title{
Weakly interacting Bose gas in a random environment
}

\author{
G. M. Falco and T. Nattermann \\ Institut für Theoretische Physik, Universität zu Köln, Zülpicher Straße 77, D-50937 Köln, Germany \\ V. L. Pokrovsky \\ Department of Physics, Texas A\&M University, College Station, Texas 77843-4242, USA \\ and Landau Institute for Theoretical Physics, Chernogolovka, Moscow District 142432, Russia \\ (Received 6 February 2009; revised manuscript received 19 June 2009; published 29 September 2009)
}

\begin{abstract}
The localization-disorder paradigm is analyzed for a specific system of weakly repulsive Bose gas at zero temperature placed into a quenched random potential. We show that at low average density or weak enough interaction the particles fill deep potential wells of the random potential whose radius and depth depend on the characteristics of the random potential and the interacting gas. The localized state is the random singlet with no long-range phase correlation. At a critical density the quantum phase transition to the coherent superfluid state proceeds. We calculate the critical density in terms of the geometrical characteristics of the noise and the gas. In a finite system the ground state becomes nonergodic at very low density. For atoms in traps four different regimes are found; only one of it is superfluid. The theory is extended to lower (one and two) dimensions. Its quantitative predictions can be checked in experiments with ultracold atomic gases and other Bose systems.
\end{abstract}

DOI: 10.1103/PhysRevB.80.104515

PACS number(s): 67.85.- d, 03.75.Hh, 03.75.Kk

\section{INTRODUCTION}

The interplay between interaction and disorder is an important paradigm of condensed-matter physics. In 1958 Anderson ${ }^{1}$ showed that in disordered solids a noninteracting electron may become localized due to the quantum interference. A phenomenological theory of localization ${ }^{2,3}$ concluded that noninteracting electrons in one and two dimensions are always localized. In three dimensions the localized and extended states are separated by the mobility edge. States with energy significantly below this edge in three dimensions are strongly localized. They appear in rare fluctuations of the quenched random potential. ${ }^{4-6}$ These instantontype states broaden and eventually overlap with growing energy. A system of noninteracting fermions in the random potential transits from the insulator to metal state when its Fermi energy exceeds the mobility edge. Thus, the Pauli principle delocalizes fermions in three dimensions, but leaves them localized in lower dimensions. The common belief is that the repulsive interaction suppresses the localization. So far this problem was studied only in the limit of a weak disorder. ${ }^{7,8}$ Therefore, the interaction induced delocalization transition remains beyond the frameworks of the theory. The metal-insulator transition in two dimensions was observed in experiments ${ }^{9}$ suggesting the decisive role of interaction.

The physical picture changes drastically for bosons. The noninteracting bosons condense at a single-particle state with the lowest energy. In a homogeneous system it leads to a coherent quantum state known as the Bose-Einstein condensate (BEC). Examples are superfluid phases of $\mathrm{He},{ }^{10}$ superconductors, ${ }^{11}$ BEC of ultracold alkali atoms, ${ }^{12,13}$ excitons in semiconductors, ${ }^{14}$ and BEC of spin waves. ${ }^{15}$ The localization of Cooper pairs was proposed as a mechanism beyond the superconductor-insulator transition. ${ }^{16} \mathrm{BEC}$ still persists when a small amount of disorder is added to the system. BEC in a random environment was observed in the superfluid phase of ${ }^{4} \mathrm{He}$ in Vycor glass or aerogels, ${ }^{17}$ in ${ }^{3} \mathrm{He}$ in aerogels, ${ }^{18}$ and in ultracold alkali atoms in disordered traps. ${ }^{19-26}$ Most of the papers concentrate on the possibility of Anderson localization in effectively one-dimensional (1D) condensates. In contrast to random potentials in crystals, the random speckle potential has a typical correlation length of the order of micron which is about of the same order as the transverse extension of the atomic cloud. The longitudinal extension is about 10 to 100 times larger. ${ }^{19}$ In most of the experiments, the expanding density profile after switching off the harmonic potential was analyzed. For weak random potential Lye et al. ${ }^{19}$ found essentially the density profile corresponding to the Thomas-Fermi theory. For larger values of the random potential these authors found a stripelike pattern of about $2 \mu \mathrm{m}$ spacing and damped dipole oscillations. For very strong disorder the atoms are localized in the minima of the random potential. Chen et al. ${ }^{24}$ also studied in situ and found fragmentation of the BEC. In some papers the superposition of a periodic plus a random potential was considered to enrich the number of possible phases. ${ }^{20,21}$ Schulte et $a .^{20}$ found features in the expanding condensate like pronounced fringes and changes in the axial size of the central peak which they trace back to the random potential.

Theoretical investigations of Bose gases or fluids in a disordered environment focused on the three following issues:

(i) the critical behavior at the normal to superfluid transition in the vicinity of the transition temperature $T_{c}(n),{ }^{27-29}$ where $n$ denotes the boson density. For fixed disorder strength, $T_{c}(n)$ decreases with decreasing boson density $n$ and eventually vanishes for some critical value of the density $n=n_{c}$.

(ii) The description of zero-temperature quantum phase transition from the superfluid to a nonsuperfluid (glassy) phase taking place at the critical value $n_{c}$ of the boson density. ${ }^{30-33}$ For a recent review of this activity see Ref. 34 .

(iii) The microscopic description of the superfluid density (and the condensate) in a disordered environment in the region of comparatively high average density of the gas $n$ $\gg n_{c}$ and $T \ll T_{c}(n) .{ }^{35-41}$ 
Since in this paper we restrict ourselves mainly to zero temperature, below we give a brief review only of the works mentioned in (ii) and (iii):

(ii) The quantum phase transition, taking place at zero temperature and $n=n_{c}$, exhibits in general a critical behavior different from that observed at finite $T_{c}(n)$. This is because in the quantum domain static and dynamic critical behavior are intertwined. This quantum phase transition was considered by $\mathrm{Ma}$ et $a l .{ }^{30}$ who mapped the problem onto a $d+1$ dimensional classical $\phi^{4}$ theory with disorder completely correlated in time. Their description gives rise to new critical exponents which were calculated by $\epsilon$ expansion around $d$ $=4$ dimensions. This work has been criticized by Fisher et $a l .{ }^{31}$ One conclusion of the work of Fisher et $a l .{ }^{31}$ is that the dynamic critical exponent $z$ should be equal to the dimension $d$ of the system in all space dimensions. This result has been disputed recently. ${ }^{32}$ Indeed, quantum Monte Carlo studies show $z \approx 1.4$ for two-dimensional (2D) system, in discrepancy with the relation $z=d .^{42}$

Some arguments of the work by Fisher et al. ${ }^{31}$ rely on the treatment of Giamarchi and Schulz ${ }^{33}$ who found the superfluid to insulator transition in one dimension. This transition happens at arbitrary disorder by increasing the interaction to a sufficiently large value. The fact that the transition into the nonsuperfluid phase happens at increasing interaction seems paradoxical, but it can be explained by the increase of quantum fluctuations of the phase. As we will show, for weak interaction there is a second transition resulting from the competition between the disorder and the interaction. We believe that namely this transition has its counterparts in higher dimension and is observed in experiments with cooled gases.

(iii) The fate of the inert layer and deeply localized (Lifshitz) states was discussed by Lee and Gunn ${ }^{35}$ without specific predictions to be checked by experiments. More recently the reduction in the superfluid and the condensate density deeply in the superfluid phase was considered microscopically by Huang and Meng. ${ }^{36}$ These authors used the Bogoliubov transformation and found a decrease in the superfluid density and of the condensate if disorder is taken into account. The corrections to both quantities are proportional to $-\left(n_{c} / n\right)^{1 / 2}$ in our terms. Thus this approach is restricted to weak disorder and $n \gg n_{c}$. These results have been confirmed and extended by other authors. ${ }^{37-41}$ All these works used the approximation of weak disorder. The extrapolation of their results to the range of strong disorder made in Ref. 36 was illegitimate.

Zhou $^{43}$ argued that the $T=0$ transition to the superfluid phase is of the percolation type. Lugan et al. ${ }^{22}$ considered an effectively one-dimensional cylindrical BEC with the disorder bounded from below but not from above. Using the numerical solution of the Gross-Pitaevskii equation as well as a mean-field-like approach they identified three different regimes: the Lifshitz glass, the fragmented BEC, and the nonfragmented BEC. Whereas their overall picture is similar to ours (when we specialize our results to the one-dimensional case), a detailed comparison is not possible since they did not present the geometrical description of the localized state. An additional discussion of this work is given in Sec. IX.

More recent papers considered the dynamic consequences of Anderson localization in BEC and do not directly overlap with our work. ${ }^{44-46}$ Using arguments similar to those used in a published work by two of the authors, ${ }^{47}$ Shklovskii ${ }^{48}$ independently and practically simultaneously arrived at the same conclusions about the critical value of density at which the transition between an insulating and the superfluid phase takes place, but he did not give the description of the localized phase.

In this paper we develop an alternative approach to the quantum phase transition starting from deeply localized state. We give a rather transparent qualitative and quantitative geometrical description of the localized state. We show that, at a critical density $n_{c}$ which we express in terms of the disorder characteristics and interaction strength, the increasing tunneling of particles between fragments leads to transition from the random singlet state to the coherent superfluid. The localized state can exist in several different regimes. These regimes as well as their quantitative characteristics strongly depend on the ratio of two basic length scales characterizing the disorder: the Larkin length $\mathcal{L}$ and the correlation length $b$. We extend our consideration to an experimentally important situation of the Bose gas confined by a harmonic trap. In this case the gas forms a cloud whose size is determined by the energy minimization. We demonstrate that all regimes which appear for the gas in the box appear also in the trap when varying the number of particles and other parameters.

Our consideration is based on theory of deeply localized single-particle states in an uncorrelated random potential given in seminal works by Lifshitz, ${ }^{4}$ Zittartz and Langer, ${ }^{5}$ and Halperin and Lax. ${ }^{6}$ Their predecessors Keldysh and Proshko $^{49}$ and Kane ${ }^{50}$ studied electron density of state in a smooth random potential having in mind semiconductors. These works were extended and detailed in Refs. 51-58. We propose a modest extension and simplification of their arguments to find some geometrical and physical characteristics of these states necessary for our purposes. On the other hand, our theory can be considered as a generalization of their instanton-type theory incorporating the self-consistent field of the interacting particles. Some preliminary results of the general theory presented here have been shown already without derivation in Ref. 59. Therefore, in order to make the paper self-contained, it was impossible to avoid some overlap with Ref. 59.

The paper is organized as follows. In the next section we characterize the random potential. In Sec. III we consider the single-particle deeply localized states in three dimensions for the uncorrelated random potential obeying the Gaussian distribution. In Sec. IV we consider how the dilute Bose gas fills the deep fluctuation potential wells in this case. In Sec. $\mathrm{V}$ the transition from the random singlet to the coherent superfluid state is considered. Section VI extends all these results to the case of strongly correlated random potential. Section VII contains a description of the Bose gas subjected to a random potential in a harmonic trap. Section VIII represents extension of these results to lower dimensions 1 and 2 . The discussion of our results and conclusions are left for Sec. IX.

\section{DESCRIPTION OF THE DISORDER}

The disorder will be represented by a random potential $U(\mathbf{x})$ with zero average at each point of the space and obeying the Gaussian distribution: 


$$
\begin{aligned}
& d W[U(\mathbf{x}), d U(\mathbf{x})] \\
& =\exp \left[-\frac{1}{2} \int_{\mathbf{x}} U(\mathbf{x}) K^{-1}\left(\mathbf{x}, \mathbf{x}^{\prime}\right) U\left(\mathbf{x}^{\prime}\right) d \mathbf{x} d \mathbf{x}^{\prime}\right] \\
& \quad \times \sqrt{\operatorname{det} K} \prod_{\mathbf{x}}(\Delta \Omega / 2 \pi)^{1 / 2} d U(\mathbf{x}),
\end{aligned}
$$

where $\Delta \Omega$ is the volume of an infinitesimal cell; $K\left(\mathbf{x}, \mathbf{x}^{\prime}\right)$ is the correlation function or correlator of the random potential

$$
K\left(\mathbf{x}, \mathbf{x}^{\prime}\right)=\left\langle U(\mathbf{x}) U\left(\mathbf{x}^{\prime}\right)\right\rangle,
$$

and $K^{-1}\left(\mathbf{x}, \mathbf{x}^{\prime}\right)$ is the inverse correlator defined by equation

$$
\int K\left(\mathbf{x}, \mathbf{x}^{\prime \prime}\right) K^{-1}\left(\mathbf{x}^{\prime \prime}, \mathbf{x}^{\prime}\right) d \mathbf{x}^{\prime \prime}=\delta\left(\mathbf{x}-\mathbf{x}^{\prime}\right) .
$$

The simplest random potential with zero correlation length has a $\delta$-like correlator:

$$
K_{0}\left(\mathbf{x}, \mathbf{x}^{\prime}\right)=\left\langle U(\mathbf{x}) U\left(\mathbf{x}^{\prime}\right)\right\rangle=\kappa^{2} \delta\left(\mathbf{x}-\mathbf{x}^{\prime}\right) .
$$

We will call it uncorrelated random potential. The corresponding distribution function is the product of independent distribution functions at each point of space:

$$
\begin{aligned}
d W_{0}[U(\mathbf{x}), d U(\mathbf{x})]= & \exp \left[-\frac{1}{2 \kappa^{2}} \int U^{2}(\mathbf{x}) d \mathbf{x}\right] \\
& \times \prod_{\mathbf{x}}\left[\left(\frac{\Delta \Omega}{2 \pi \kappa^{2}}\right)^{1 / 2} d U(\mathbf{x})\right] .
\end{aligned}
$$

More general Gaussian random potential has a finite correlation length $b$. The simplest realization of the random Gaussian potential with the finite correlation length is the OrnsteinZernike (OZ) correlator:

$$
K_{\mathrm{OZ}}\left(\mathbf{x}, \mathbf{x}^{\prime}\right)=\frac{\kappa^{2}}{4 \pi b^{2}} \frac{\exp \left(-\frac{\left|\mathbf{x}-\mathbf{x}^{\prime}\right|}{b}\right)}{\left|\mathbf{x}-\mathbf{x}^{\prime}\right|} .
$$

The corresponding probability distribution reads

$$
\begin{aligned}
& d W_{\mathrm{OZ}} {[U(\mathbf{x}), d U(\mathbf{x})] } \\
&= \exp \left\{-\frac{1}{2 \kappa^{2}} \int\left[U^{2}(\mathbf{x})+b^{2}(\nabla U(\mathbf{x}))^{2}\right] d \mathbf{x}\right\} \\
& \times \prod_{\mathbf{q}}\left\{\left[\frac{\left(1+b^{2} q^{2}\right)}{2 \pi \kappa^{2}} \Delta \Omega_{\mathbf{q}}\right]^{1 / 2} d \tilde{U}(\mathbf{q})\right\},
\end{aligned}
$$

where $\tilde{U}(\mathbf{q})$ is the Fourier transformation of $U(\mathbf{x})$ and $\Delta \Omega_{\mathbf{q}}$ is the element of volume in the momentum space. At $b=0$ Eq. (7) turns into Eq. (5). The OZ distribution has a characteristic energy scale $U_{0}=\kappa / b^{3 / 2}$.

The disorder correlator may be regular at coincident coordinates $\mathbf{x}=\mathbf{x}^{\prime}$. Then it has the following form:

$$
K\left(\mathbf{x}, \mathbf{x}^{\prime}\right)=\left\langle U^{2}\right\rangle h\left(\frac{\left|\mathbf{x}-\mathbf{x}^{\prime}\right|}{b}\right),
$$

where $\left\langle U^{2}\right\rangle \equiv\left\langle U^{2}(\mathbf{x})\right\rangle$ is the average quadratic fluctuation of the random field at a point. The function $h(u)$ which determines the shape of the correlation function is normalized by the condition $h(0)=1$. In this case the Fourier component of the inverse correlator is a growing function of the wave vector. As a consequence, $K^{-1}\left(\mathbf{x}, \mathbf{x}^{\prime}\right)$ is not well defined. Instead we can use the probability in the momentum space:

$$
\begin{aligned}
d W[\tilde{U}(\mathbf{q}), d \tilde{U}(\mathbf{q})]= & \exp \left(-\int \frac{\tilde{U}(\mathbf{q}) \tilde{U}(-\mathbf{q})}{2 \tilde{K}(\mathbf{q})} d \mathbf{q}\right) \\
& \times \prod_{\mathbf{q}}\left(\frac{\Delta \Omega_{\mathbf{q}}}{2 \pi \tilde{K}(\mathbf{q})}\right)^{1 / 2} d \tilde{U}(\mathbf{q}) .
\end{aligned}
$$

Simple examples of nonsingular correlators are the Lorenz distribution with $h_{L}(u)=\left(u^{2}+1\right)^{-1}$ and $\tilde{K}_{L}(\mathbf{q})$ $=2 \pi^{2}\left\langle U^{2}\right\rangle b^{2} q^{-1} \exp (-b q)$ and the Gaussian distribution with $h_{G}(u)=\exp \left(-u^{2} / 2\right)$ and $\widetilde{K}_{G}(\mathbf{q})=(2 \pi)^{3 / 2}\left\langle U^{2}\right\rangle b^{3} \exp \left(-b^{2} q^{2} / 2\right)$. In all these cases the scale of energy is established by $U_{0}$ $=\sqrt{\left\langle U^{2}\right\rangle}$ and the scale of length is $b$.

\section{SINGLE-PARTICLE LEVELS IN AN UNCORRELATED RANDOM POTENTIAL}

In this section we study the properties of single-particle states in the uncorrelated random potential defined by Eqs. (4) and (5) and illustrated by Fig. 1. This is a modest extension of the well-known works in Refs. 4-6. Some simplifications allow us to find more detailed information about the distribution functions of the energy, sizes, and distances between the states and the tunneling amplitudes between them. We are interested in a statistical description of the spectrum and wave functions of Schrödinger equation in the random potential $U(\mathbf{x})$ :

$$
\frac{\hbar^{2}}{2 m} \nabla^{2} \psi+[E-U(\mathbf{x})] \psi=0 .
$$

Its energy levels $E[U(\mathbf{x})]$ in a finite volume are functionals of the potential $U(\mathbf{x})$. The only characteristic of the random potential $\kappa$ together with world constants $\hbar$ and $m$ establishes the scale of length

$$
\mathcal{L}=\frac{\hbar^{4}}{m^{2} \kappa^{2}},
$$

which will be called Larkin length in analogy with the scale found in Larkin's work ${ }^{60}$ for an elastic medium in a random field. We will show that $\mathcal{L}$ sets the scale of the extension of the deeply localized states with large by modulus negative energy. For delocalized states with large positive energy the same value with precision of numerical factor is the mean free path. The scale of energy $\mathcal{E}$ can be obtained from the Larkin length in an obvious way:

$$
\mathcal{E}=\frac{\hbar^{2}}{m \mathcal{L}^{2}}=\frac{m^{3} \kappa^{4}}{\hbar^{6}} .
$$

Further, we will work in a rather rough approximation similar to that used by Larkin and Ovchinnikov ${ }^{61}$ and Imry and Ma. ${ }^{62}$ However, we start with a rigorous statement of the problem which gives a clue for our further estimates. The most easily calculable value is the density of state $\nu(E, \Omega)$ 


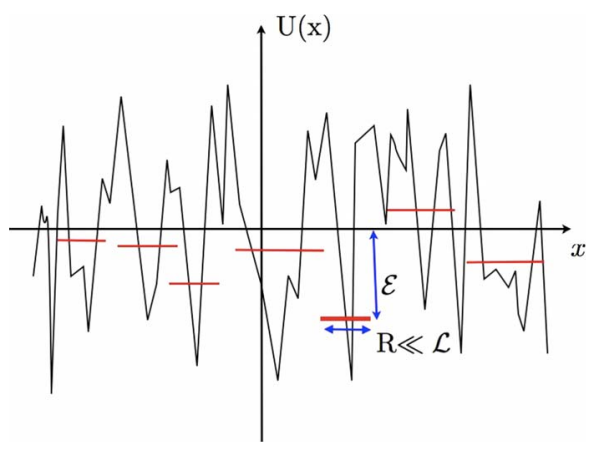

FIG. 1. (Color online) Uncorrelated potential, the characteristic length, and energy scales are $\mathcal{L}$ and $\mathcal{E}$, respectively. There is typically only one bound state in a single potential well.

which can be written as a path integral (see the cited works $\left.^{4-6}\right)$ :

$$
\nu(E, \Omega)=\frac{1}{\Omega} \int \delta(E-E[U(\mathbf{x})]) d W[U(\mathbf{x}), \Omega],
$$

where $E[U(\mathbf{x})]$ is the spectrum of eigenvalues of the Schrödinger Eq. (10) in the volume $\Omega$.

In a large three-dimensional (3D) volume the states with energy $E \gg \mathcal{E}$ are delocalized, whereas the states with negative energy sufficiently large by modulus $E<0$ and $|E| \gg \mathcal{E}$ are strongly localized. The threshold of localization is a positive energy of the order of $\mathcal{E}$. Note that this is correct only for 3D systems. As it was conjectured in the work by Abrahams et al., ${ }^{2}$ all single-particle states in $1 \mathrm{D}$ and 2D systems are localized. Near the threshold the wave function has very complicated fractal structure. ${ }^{63,64}$ In the interval between $\mathcal{E}$ and $-\mathcal{E}$ the transition from fractal to strongly localized states evolves. The latter are supported by rare fluctuations of the random potential, which form a potential well sufficiently deep to have the negative energy $E$ as its only bound state. The problem of the deep random levels was considered by already mentioned authors ${ }^{4-6}$ about 40 years ago. We reproduce some of their results and extend them to find the probability distribution of the levels with energy less than some $E$ $(E<0 ;|E| \gg \mathcal{E})$, the distances between such states and the tunneling amplitude between them.

As it is clearly seen from Eqs. (1) and (13), the main exponential factor in the density of state can be found by minimization of the integral $\int_{\Omega} U^{2}(\mathbf{x}) d \Omega$ at a fixed value of the energy level $E[U(\mathbf{x})]$, which is a functional of the random potential $U(\mathbf{x})$. The latter can be determined as a minimum of the energy over the wave function

$$
\begin{aligned}
E[U(\mathbf{x})] & =\min _{\psi(\mathbf{x})} E[U(\mathbf{x}), \psi(\mathbf{x})] \\
& =\min _{\psi(\mathbf{x})} \int\left[\frac{\hbar^{2}}{2 m}|\nabla \psi(\mathbf{x})|^{2}+U(\mathbf{x})|\psi(\mathbf{x})|^{2}\right] d \Omega .
\end{aligned}
$$

Thus, we need to minimize a functional,

$$
\begin{aligned}
F[U(\mathbf{x}), \psi(\mathbf{x})]= & \int U^{2}(\mathbf{x}) d \Omega-\lambda \int\left[\frac{\hbar^{2}}{2 m}|\nabla \psi(\mathbf{x})|^{2}\right. \\
& \left.+U(\mathbf{x})|\psi(\mathbf{x})|^{2}\right] d \Omega,
\end{aligned}
$$

over $\psi(\mathbf{x})$ and $U(\mathbf{x})$. Here $\lambda$ is a Lagrangian factor. The minimization over $\psi(\mathbf{x})$ leads to Schrödinger Eq. (10), whereas the minimization over $U(\mathbf{x})$ results in a relation between $U(\mathbf{x})$ and $\psi(\mathbf{x})$ :

$$
U(\mathbf{x})=\lambda|\psi(\mathbf{x})|^{2} .
$$

Thus, Eq. (10) turns into the Ginzburg-Landau equation. For our purpose the most important consequence of relationship (16) is that the fluctuation potential well $U(\mathbf{x})$ has the same characteristic linear size $R$ as the wave function $\psi(\mathbf{x})$. It is clear that the maximum probability requires the bound state with the fixed energy $E$ to be the only bound state in the potential well. Otherwise, at the same energy, we need a deeper well, i.e., larger $U^{2}(\mathbf{x})$. For the same reason the fluctuation well must have the spherical shape. Let the radius of the well is $R$. Then the depth of the well can be estimated as $U_{\min } \sim-\hbar^{2} / m R^{2}$ and the energy level in it differs by a factor of the order of 1/2: $E \sim-\hbar^{2} / 2 m R^{2}$. The exponential factor in the density of state reads

$$
\exp \left(-\frac{\frac{4 \pi}{3} R^{3} U^{2}}{2 \kappa^{2}}\right)=\exp \left(-\frac{\mathcal{L}}{R}\right)=\exp \left[-\left(\frac{|E|}{\mathcal{E}}\right)^{1 / 2}\right],
$$

where we redefined the Larkin length $\mathcal{L}$ incorporating the factor $2 \pi / 3$ in it. Here and henceforth we perform calculations for 3D systems. The results for other dimensions will be derived later. Result (17) is valid provided the number in the exponent is large, i.e., $R \ll \mathcal{L}$ and $|E| \gg \mathcal{E}$.

Let us consider the probability or the part of volume $q(R)$ occupied by the wells with the radius less than $R$ or the energy less than $E=-\hbar^{2} / 2 m R^{2}$. It is obvious that $q(R)$ contains an exponential factor (17) and some preexponent. Since $q(R)$ is a dimensionless value, the preexponent must be a function of the dimensionless ratio $\mathcal{L} / R$ :

$$
q(R)=f\left(\frac{\mathcal{L}}{R}\right) \exp \left(-\frac{\mathcal{L}}{R}\right) .
$$

The function $f(x)$ must be much slower function of its argument than the exponent. Most naturally it is a power function $f(x) \sim x^{\alpha}$ with a critical exponent $\alpha$. It can be extracted from the work by Cardy: ${ }^{51} \alpha=1$ for the uncorrelated disorder. We will see that it is inessential for further conclusions. By knowledge of $q(R)$ we can calculate the number $n_{w}(R)$ of the wells with the radius less than $R$ per unit volume, i.e., the density of such wells. ${ }^{65}$ In order to do that the unit volume must be divided into the cells of the volume $R^{3}$. Each cell can contain or do not contain the fluctuation, but fluctuations with the centers approaching each other at a distance less than $R$ must be considered as one asymmetric potential well. The number of such cells in unit volume is $R^{-3}$. Thus, 


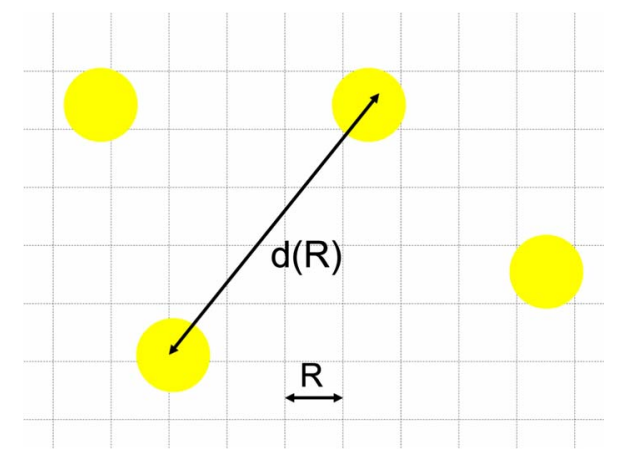

FIG. 2. (Color online) Deeply localized states. For low densities of bosons the distances between deeply localized states of radius $R \ll \mathcal{L}$ (or smaller) are separated by distances $d(R) \gg \mathcal{L}$.

$$
n_{w}(R)=R^{-3} q(R)=R^{-3} f\left(\frac{\mathcal{L}}{R}\right) \exp \left(-\frac{\mathcal{L}}{R}\right) .
$$

The average distance $d(R)$ between the wells with the radius less than $R$ reads

$$
d(R)=\left[n_{w}(R)\right]^{-1 / 3}=R f^{1 / 3} \exp \left(\frac{\mathcal{L}}{3 R}\right) .
$$

This equation shows that the distance between the wells is significantly larger than the size of the wells. The situation is depicted in Fig. 2. Finally we find the tunneling factor $t(R)$ for two typical wells with the energy levels of the same order or the radius $R$ of the same order of magnitude. It is given by the semiclassical expression $t(R)=\exp \left(-\frac{1}{\hbar} \int|p| d l\right)$, where the integral path connects two wells. By the order of magnitude $|p| \sim \sqrt{2 m|E|} \sim \hbar / R$ and the length of the path of integration is $\sim d(R)$. Thus, $\frac{1}{\hbar} \int|p| d l \sim d / R=f^{1 / 3} \exp \left(\frac{\mathcal{L}}{3 R}\right)$. Finally, we find

$$
t(R)=\exp \left[-f^{1 / 3} \exp \left(\frac{\mathcal{L}}{3 R}\right)\right]
$$

At $R \sim \mathcal{L} / 3$ or $E \sim-9 \mathcal{E}$, the distances between the optimal potential wells become of the same order of magnitude as their size $R$. Simultaneously the tunneling amplitude between the wells becomes of the order of 1 . The potential wells percolate and tunneling is not small, but the states still are not propagating due to the Anderson localization. ${ }^{1}$

\section{BOSE GAS IN A LARGE BOX WITH AN UNCORRELATED RANDOM POTENTIAL}

In the ground state of an ideal Bose gas in a large box with the Gaussian random potential all particles are located at the deepest fluctuation level. In the box of the cubic shape with the side $L$ the deepest level which occurs with probability of the order of 1 has the radius $R$ determined by equation: $L^{3} n_{w}(R)=1$, i.e., $R \sim \frac{\mathcal{L}}{3 \ln (L / \mathcal{L})}$. The prefactor introduces a correction to the denominator of the order of $\ln \left(\ln \frac{L}{\mathcal{L}}\right)$ which can be neglected. The corresponding energy is $E \sim-9 \mathcal{E}\left(\ln \frac{L}{\mathcal{L}}\right)^{2}$. Such a state is highly nonergodic since the location and the depth of the deepest level strongly depend on a specific realization of the disordered potential. Therefore, the average energy per particle and other properties averaged over the ensemble have nothing in common with the properties of a specific sample. Even an infinitely small interaction immediately changes the situation: the system becomes ergodic in the thermodynamic limit, i.e., when first the size of the system grows to infinity and then the interaction goes to zero. For example, the energy per particle in sufficiently large volume coincides with its average over the ensemble. The reason of such a sharp change is that, at any small but finite interaction, the particles cannot more fill one well since their repulsion finally overcomes the attraction to the potential well. They will be redistributed over multiple wells. Since the distribution of wells in different parts of sufficiently large volume passes all possible random configurations with proper ensemble probabilities, the ergodicity is established. In this section we find how the interacting particles eventually fill deep single-particle levels simultaneously changing their size and shape by their self-consistent field.

In a real experiment the Bose gas may be quenched in a metastable state depending on the cooling rate and other nonthermodynamic factors. This is what Fisher et al. ${ }^{31}$ call the Bose glass. Such a state is also possible in the case of weakly repulsive Bose gas. It looks plausible that the Bose glass whose metastable state has energy close to that of the ground state has also close to the ground state statistical characteristics, but this question needs more thorough study. However, as it will be demonstrated later, in the case of cooled alkali atoms the tunneling amplitude still remains large enough to ensure the relaxation to the equilibrium state in $10^{-3}-10^{-2} \mathrm{~s}$. Our further estimates relate to the real ground state.

The weakly repulsive Bose gas in a random potential is described by the well-known Hamiltonian

$$
H=\sum_{\mathbf{p}} \frac{\mathbf{p}^{2}}{2 m} a_{\mathbf{p}}^{\dagger} a_{\mathbf{p}}+\frac{g}{2} \int\left(\psi^{\dagger} \psi\right)^{2} d \mathbf{x}+\int U(\mathbf{x}) \psi^{\dagger} \psi d \mathbf{x},
$$

where $\psi(\mathbf{x})=\Omega^{-1 / 2} \Sigma_{\mathbf{p}} a_{\mathbf{p}} \exp (i \mathbf{p x} / \hbar)$ is the secondary quantized wave function; the positive coupling constant $g$ is associated with the scattering amplitude $a$ by the relationship ${ }^{66}$

$$
g=\frac{4 \pi \hbar^{2} a}{m}
$$

As in Bogoliubov's theory ${ }^{67}$ and its extension by Belyaev, ${ }^{68}$ we assume that the gas criterion $n a^{3} \ll 1$ is satisfied. Here $n$ $=N / \Omega$ is the average particle density and $N$ is their total number. The purpose of the following consideration is to find how, with the growth of the number of particles, they eventually fill the fluctuation potential wells. Implicitly our considerations take into account the change in the optimal potential wells due to the interaction.

Let $N$ particles with the average density of particles $n$ fill all potential wells with the radii less than $R$ in the ground state. The average number of particles per well is

$$
\mathcal{N}(R)=\frac{n}{n_{w}(R)} .
$$

The local density inside the well of the linear size of $R$ is $n_{p}(R)=3 \mathcal{N}(R) / 4 \pi R^{3}$. The gain of energy per particle due to the random potential is $E(R)=-\hbar^{2} / 2 m R^{2}$; the repulsion energy due to interaction is equal to $g n_{p}(R)=3 \hbar^{2} \mathcal{N}(R) a / m R^{3}$, 
where we used Eq. (23) and the well-known relation for an effective potential field induced by a gas of scatterers. ${ }^{66}$ Minimizing the total energy per particle

$$
E_{\mathrm{tot}}(R)=-\frac{\hbar^{2}}{2 m R^{2}}+\frac{3 \hbar^{2} \mathcal{N}(R) a}{m R^{3}}
$$

over $R$ and employing Eqs. (19) and (24), we find the value of $R$ corresponding to the minimum of energy at fixed $n$ with the logarithmic precision:

$$
R(n)=\frac{\mathcal{L}}{\ln \frac{n_{c}}{n}},
$$

where the critical density $n_{c}$ is defined as follows:

$$
n_{c}=\left(3 \mathcal{L}^{2} a\right)^{-1} \text {. }
$$

The critical density can be treated as the density at which the average attraction energy $\mathcal{E}$ of the random potential equals to the energy per particle $n g$. The factor $f$ in Eq. (19) as well as the next approximation to solution (26) leads to the corrections of the type $\ln \left(\ln \frac{n_{c}}{n}\right)$ which can be neglected. The distances between the filled wells according to Eq. (20) are

$$
d(n)=\frac{\mathcal{L}}{\ln \frac{n_{c}}{n}}\left(\frac{n_{c}}{n}\right)^{1 / 3} .
$$

This distance strongly exceeds the average size of potential well (26) at $n \ll n_{c}$. At the same condition the chemical potential of atoms can be estimated as

$$
\mu(n)=-\frac{\hbar^{2}}{2 m R^{2}(n)}=-\frac{\mathcal{E}}{2}\left(\ln \frac{n_{c}}{n}\right)^{2} .
$$

The tunneling amplitude $t(n)$ between two wells separated by a typical distance $d(n)$ can be found by employing the singleparticle result $(21)$ :

$$
t(n)=\exp \left[-f\left(\frac{n_{c}}{n}\right)^{1 / 3}\right],
$$

where $f \equiv f\left(\ln \frac{n_{c}}{n}\right)$. Since $f(x)$ has a powerlike asymptotic at large $x$, one finds $f \sim\left(\ln \frac{n_{c}}{n}\right)^{\alpha}$.

Thus, the Bose gas at $n \ll n_{c}$ is fragmented into multiple clusters of small size $R(n)$ separated by much larger distances $d(n)$ and containing about $\mathcal{L} /\left[3 a\left(\ln \frac{n_{c}}{n}\right)^{3}\right]$ particles each. The amplitude of tunneling between the wells is exponentially small, so that the number of particles in each cluster is well defined. The phase is therefore completely uncertain. Such a state is a singlet with inhomogeneously distributed particles, a random singlet: the ground state is nondegenerate.

Let us outline a more quantitative approach to the same problem which allows to find the exact shape of the optimal fluctuation and exact figure in the exponent. Let $\mathcal{N}$ particles fill a random potential well with the potential $U(\mathbf{r})$. The effective potential acting on a particle is $U_{\text {eff }}(\mathbf{r})=U(\mathbf{r})$ $+g n_{p}(\mathbf{r})$, where $n_{p}(\mathbf{r})$ is the coordinate-dependent density of particles obeying the constraint $\int n_{p}(\mathbf{r}) d^{3} \mathbf{r}=\mathcal{N}$. We assume that the self-consistent potential well $U_{\text {eff }}(\mathbf{r})$ has only one bound state and all $\mathcal{N}$ particles occupy it. Then the particle density $n_{p}(\mathbf{r})$ is related to the normalized wave function of this state $\psi(\mathbf{r})$ by the following equality: $n_{p}(\mathbf{r})=\mathcal{N}\left|\psi^{2}(\mathbf{r})\right|$. Similarly to the single-particle case, the most probable fluctuation realizes the minimum of the functional:

$$
\begin{aligned}
F(U, \psi)= & \frac{1}{2} \int U^{2}(\mathbf{r}) d^{3} \mathbf{r}-\lambda \int\left\{\frac{\hbar^{2}}{2 m}|\nabla \psi|^{2}\right. \\
& \left.+\left[U_{\text {eff }}(\mathbf{r})-E\right]\left|\psi^{2}(\mathbf{r})\right|\right\} d^{3} \mathbf{r} .
\end{aligned}
$$

The minimization proceeds over $\psi$ and $U$ independently at a fixed $n_{p}(\mathbf{r})$. The minimization over $U$ results again in the relationship $U=\lambda\left|\psi^{2}\right|$. Therefore, the effective potential $U_{\text {eff }}(\mathbf{r})$ is also proportional to $\left|\psi^{2}\right|: U_{\text {eff }}(\mathbf{r})=-\Lambda\left|\psi^{2}(\mathbf{r})\right|$, where $\Lambda=\lambda-g \mathcal{N}$. Thus, the wave function $\psi$ satisfies the GinzburgLandau (nonlinear Schrödinger) equation

$$
\nabla^{2} \psi-q^{2} \psi-l \psi^{3}=0,
$$

where $q^{2}=-2 m E / \hbar^{2}$ and $l=2 m \Lambda / \hbar^{2}$. By a scaling transformation $\mathbf{r}=\alpha \mathbf{x}, \psi=\beta \phi$ Eq. (32) can be reduced to a standard form,

$$
\nabla^{2} \phi-\phi^{3}=\varepsilon \phi
$$

with the normalization condition $\int \phi^{2} d^{3} x=1$. These requirements determine all unknown values: $\alpha=\sqrt{\varepsilon} / q ; \beta=\alpha^{-3 / 2}$ and $l=\alpha$. The argument of exponent in the expression for configuration probability (5) thus reads

$$
\Phi=\frac{\lambda^{2} \alpha^{3} \beta^{4}}{2 \kappa^{2}} \phi_{4}=\frac{\hbar^{4} q(1+\gamma)^{2}}{8 m^{2} \kappa^{2} \sqrt{\varepsilon}} \phi_{4},
$$

where $\gamma=g \mathcal{N} / \Lambda$ and $\phi_{4}=\int \phi^{4} d^{3} x$. By definition $q=1 / R$. Therefore, expression (34) can be rewritten as $\Phi=C \frac{\mathcal{L}}{R}(1$ $+\gamma)^{2}$ with numerical coefficient $C=\frac{3}{16 \pi \sqrt{\varepsilon}} \int \phi^{4} d^{3} x$. The eigenvalue $\varepsilon$ and $\phi_{4}$ for the normalized solution of Eq. (33) can be calculated numerically. We find $\varepsilon \sim 353.082$ and $\phi_{4}$ $\sim 1420.35$. ${ }^{69}$ With these values we find $C=4.50$. To find $\mathcal{N}$ we apply a rough approximation for the number of particles per unit volume: $n=\mathcal{N} R^{-3} \exp (-\Phi)$. From this equation we find with the logarithmic precision:

$$
\ln \frac{\mathcal{N}}{n \mathcal{L}^{3}}=C \frac{\mathcal{L}}{R}(1+\gamma)^{2} .
$$

This equation can be treated as an implicit equation for the maximal radius of filled potential wells $R$ as function of $\mathcal{N}$. The value $\mathcal{N}$ must minimize energy or equivalently $R$. Taking derivatives of both sides of Eq. (35) by $\mathcal{N}$ and assuming $\frac{d R}{d \mathcal{N}}=0$, one finds

$$
\mathcal{N} \ln \frac{\mathcal{N}}{n \mathcal{L}^{3}}=\frac{\Lambda(1+\gamma)}{2 g}=\frac{R(1+\gamma) \sqrt{\varepsilon}}{16 \pi C a} .
$$

From Eqs. (35) and (36) it is possible to find $\mathcal{N}$ and $R$. With the same logarithmic precision they are 


$$
R=\frac{C \mathcal{L}}{\ln \frac{n_{c}}{n}}, \quad \mathcal{N}=\frac{\mathcal{L}}{a} \frac{\sqrt{\varepsilon}}{16 \pi \ln ^{2} \frac{n}{n_{c}}}
$$

in good agreement with our simple estimates. The value $\gamma$ is logarithmically small: $\gamma \approx\left(\ln \frac{n_{c}}{n}\right)^{-1}$. Further, we apply our rough estimates.

The previous approach is valid if $\mathcal{N}(R) \gg 1$. From $\mathcal{N}(R)$ found in the previous paragraph we conclude that the disorder must be weak enough to satisfy the constraint $\mathcal{L} \gg 3 a$. The latter inequality is necessary but not sufficient. Additionally the average density $n$ must be large enough. Namely, it must satisfy strong inequalities $\exp \left[-\left(\frac{\mathcal{L}}{3 a}\right)^{1 / 2}\right] n_{c} \ll n \ll n_{c}$. At smaller $n$, only few particles appear in a potential well. At $n \ll \exp \left[-\left(\frac{\mathcal{L}}{3 a}\right)^{1 / 2}\right] n_{c}$, the size of filled potential wells approaches $36 \mathrm{a}$. Most of the filled wells contain two particles, but only a small part of the total number of wells of this size (per unit volume) $n_{w}(36 a)$ is filled, approximately 0.5 $\times 10^{-4} a^{-3} \exp \left(-\frac{\mathcal{L}}{36 a}\right)$. The consistency of our approximation requires that the gas parameter remains small inside an optimal potential well. The density inside the well is equal to $n_{c}$. Therefore, the interaction is weak inside the well if $n_{c} a^{3}$ $=\frac{a}{3 \mathcal{L}} \ll 1$.

The compressibility $\partial n / \partial \mu=\frac{n}{\mathcal{E}} \ln \left(\frac{n_{c}}{n}\right)$ is finite as expected for the Bose glass phase. ${ }^{31}$ In the absence of interaction the probability that two single-particle eigenstates localized in different potential wells have the same energy is zero. Thus, the zero-temperature conductivity vanishes in the thermodynamic limit. If the interaction is switched on, the energy levels split by an amount of the order $g n_{p}$. For $n \ll n_{c}$ the wave functions are still localized and the conductivity at zero temperature vanishes. Thus, the Bose gas in the ground state or in the Bose glass is insulating, in the meaning that external volume force (the gravitational field) does not cause a macroscopic flow. At finite temperatures, however, the energy gap between different localized states can be bridged by thermal activation leading to the Mott variable range hopping (VRH). ${ }^{58}$ Below we apply the VRH argument to our situation. The tunneling probability between two wells, each of extension $R$, separated by the distance $L$, is $|t(L, R)|$ $=\exp (-2 L / R)$ (see analogous derivation in Sec. III). Together with the activation factor it gives the hopping probability between the two wells equal to

$$
P(T) \sim e^{-2 L / R} e^{-\Delta E / T},
$$

where $\Delta E$ is the energy difference between the two distant wells. There is with high probability at least one localized state in the interval of energy $\Delta E$ provided the condition $\Delta E \nu(E) L^{3} \gtrsim 1$ is satisfied. Employing the derived dependencies $E \approx-\hbar^{2} /\left(2 m R^{2}\right), \quad R=\mathcal{L} / \ln \left(n_{c} / n\right)$, and $\nu(E)$ $=\mathcal{E}^{-1} R^{-3} \exp (-\mathcal{L} / R)$ and maximizing probability (38) over $L$, we find the hopping conductivity $\sigma(T)$,

$$
\sigma(T) \sim e^{-C\left[\mathcal{E} n_{c} /(T n)\right]^{1 / 4}},
$$

where $C$ is a constant or slow function of $\ln n_{c} / n$. In a similar way it is possible to calculate the nonlinear fielddependent conductivity.
At the VRH thermal excitations (phonons, spin waves, etc.) must be absorbed. In the diluted Bose gas these excitations could be phonons. However, in the localized state the gas is separated in fragments. The oscillations of density in a fragment have the energy gap of the order $\sqrt{4 \pi n_{c} a} \hbar^{2} /(m \mathcal{L})$ $\sim \mathcal{E}$. There are also oscillations of the entire cloud volume whose frequency is determined by the cloud side and the above calculated compressibility. It is not clear, however, what the density of states is of these phonons. They definitely attenuate strongly when their wavelength becomes of the order or shorter than the distances between potential wells $d(n)$. Thus, we cannot reliably use the VRH results to the cooled gases. However, they are reasonable for condensates realized in solids: the exciton and spin-wave condensates.

\section{TRANSITION REGION}

Though our results are valid only for $n \ll n_{c}$, it follows from them that at $n \sim n_{c}$ the overlapping of different occupied wells becomes large and the tunneling amplitudes reach the value of the order of 1 . Therefore, the phase coherence in different wells grows until at some critical value of density which we roughly identify with $n_{c}$ the global coherence is established. Thus, at $n \approx n_{c}$, the quantum phase transition from localized singlet to the superfluid state proceeds. As usual, the order parameter is the average phase factor $\langle\exp (i \varphi)\rangle$. The superfluid state bears clear traces of disorder: its superfluid density is inhomogeneous at characteristic scale $\mathcal{L}$. It can be treated as a disordered superfluid. An alternative argument goes as follows. The condition $n \approx n_{c}$ can be rewritten in the form $\mathcal{L} \approx \xi$ where $\xi=(a n)^{-1 / 2}$ denotes the superfluid healing length. The latter describes the range over which the superfluid order parameter, when changed by a local perturbation (e.g., by a wall or in the center of a vortex), regains its bulk value. Since $\mathcal{L}$ denotes the scale at which the disorder becomes relevant, the condition $\xi \gg \mathcal{L}$ simply means that the superfluid order parameter cannot adapt to the rapid variation in the disorder which happens at the scale $\mathcal{L}$ and superfluidity is destroyed. In the opposite case $n \gg n_{c}$, i.e., $\xi \ll \mathcal{L}$ this is not longer the case and superfluidity survives.

At $n \gg n_{c}$, the energy of the repulsion $g n$ becomes much larger than the characteristic disorder energy $\mathcal{E}$. Then the gas becomes almost homogeneous with precision of small parameter $\mathcal{E} / g n \sim n_{c} / n$. In this case the approach by Huang and Meng $^{36}$ based on the homogeneous zero-approximation ground state and refined by Giorgini et al. ${ }^{37}$ and by Lopatin and Vinokur ${ }^{38}$ is justified. They demonstrated that the superfluid density $n_{s}$ is not equal to the total density as it is without disorder, but remains close to the total density. Thus, the superfluidity definitely strives in the limit of large density. We conjecture that $n_{s}$ vanishes at the same quantum phase transition point $n=n_{c}$ at which the coherence disappears.

The small corrections found in the cited work ${ }^{36}$ can be estimated by the order of magnitude without long calculations. Indeed, the disorder Hamiltonian reads

$$
H_{\text {dis }}=\sum_{\mathbf{p}, \mathbf{q}} U_{\mathbf{q}} a_{\mathbf{p}}^{\dagger} a_{\mathbf{p}+\mathbf{q}},
$$

where $U_{\mathbf{q}}$ is Fourier transform of the random field $U(\mathbf{x})$. It produces the change in energy in the second order of the 
perturbation theory. The change in energy per particle can be estimated as follows:

$$
\Delta \varepsilon=\int \frac{\left\langle U_{\mathbf{q}}^{2}\right\rangle}{\varepsilon_{\mathbf{q}}} \frac{d^{3} q}{(2 \pi \hbar)^{3}} .
$$

The deltalike correlation function of random potential (4) implies that $\left\langle U_{\mathbf{q}}^{2}\right\rangle=\kappa^{2}$ for any $\mathbf{q}$. The excitation energy $\varepsilon_{\mathbf{q}}$ can be estimated as $\sqrt{\frac{g n}{m}} q$ in the effective range of integration $q$ $<\sqrt{m g n}$ after subtraction of the contribution of disorder to the energy of the normal state. Collecting all these factors, we find

$$
\Delta \varepsilon \approx \frac{\kappa^{2} m^{3 / 2}(g n)^{1 / 2}}{4 \pi^{2} \hbar^{3}}=\frac{\kappa^{2}(m a n)^{1 / 2}}{2 \sqrt{\pi} \hbar^{2}} .
$$

With precision of a numerical coefficient 0.85 this result coincides with that obtained by Huang and Meng [Eq. (7) of their work $^{36}$ gives the value of energy per unit volume; it must be divided by $n$ to find the energy of the ground state per particle]. In terms of the Larkin length and energy and critical density introduced earlier [see Eqs. (11), (12), and (27)] the correction to the energy of the ground state per particle can be expressed as

$$
\Delta \varepsilon \approx \frac{\sqrt{\pi}}{3} \mathcal{E}\left(\frac{n}{n_{c}}\right)^{1 / 2} \sim \frac{\hbar^{2}}{m \xi^{2}} \frac{\xi}{\mathcal{L}} .
$$

The change in superfluid and condensate densities can be found from their initial values (one exactly and another approximately equal to the total density $n$ ) by multiplication to a small factor proportional to $\Delta \varepsilon / \varepsilon_{0} \sim \xi / \mathcal{L}$, where $\varepsilon_{0}$ $=g n / 2=2 \pi \hbar^{2} a n / m \sim \hbar^{2} / m \xi^{2}$.

\section{CORRELATED DISORDER}

In this section we consider properties of the weakly interacting Bose gas in a big box with a correlated random potential. The properties of correlated random potential were already described in the second half of Sec. II [see the text related to Eqs. (8) and (9)]. Here, following the line of consideration developed in Secs. III-V, we study first the size and distribution of single-particle states and tunneling between them and how they are modified by interaction. We start with the single-particle states.

\section{A. Single-particle states in a correlated random potential}

Keldysh and Proshko, ${ }^{49}$ and Kane ${ }^{50}$ were the first to find the electron density of states in a semiclassical random potential. They proved that, in contrast to the uncorrelated disorder for which $\nu(E) \sim \exp \left[-(E / \mathcal{E})^{1 / 2}\right]$, the density of states in a correlated disorder is a Gaussian function of energy $\nu(E) \sim \exp \left[-c\left(E / U_{0}\right)^{2}\right]$, where $c$ is a numerical constant. Independently but later the same result was found in Refs. 52-54. Shklovskii and Efros ${ }^{55}$ derived the same result employing the instanton approach. John and Stephen ${ }^{56}$ found the dependence of the preexponential factor on energy. Their result was confirmed by Thirumalai ${ }^{57}$ by a different method. Below we rederive their result and find important characteristics of optimal fluctuation wells in the case when the cor- relation length is large enough. It is intuitively clear that, at $b \ll \mathcal{L}$, the density of states and other characteristics of the spectrum and optional states only slightly differ from their values for uncorrelated random potential. At $b \sim \mathcal{L}$ numerical coefficients will be different from those for uncorrelated disorder, but with this reservation still our semiquantitative description is valid. Therefore, the most interesting is the situation with $b \gg \mathcal{L}$. Such a disorder we call strongly correlated. In what follows we assume the strongly correlated disorder. The Larkin length for the correlated disorder reads

$$
\mathcal{L}=\frac{3 \hbar^{4}}{4 \pi m^{2} U_{0}^{2} b^{3}}
$$

It is convenient to introduce another characteristic length $B$ $=(3 / 4 \pi)^{1 / 4}\left(\hbar^{2} / m U_{0}\right)^{1 / 2}$. For strongly correlated disorder $B$ $\ll b$. The Larkin length is associated with $B$ by the following relation:

$$
\frac{\mathcal{L}}{b}=\left(\frac{B}{b}\right)^{4} \text {. }
$$

To find the exponent in the density of deeply localized states it is necessary to minimize the functional $\int U(\mathbf{x}) K^{-1}\left(\mathbf{x}, \mathbf{x}^{\prime}\right) U\left(\mathbf{x}^{\prime}\right) d \mathbf{x} d \mathbf{x}^{\prime}$ at fixed energy of the quantum state in the potential $U(\mathbf{x})$. The condition of minimum is reduced to a following relationship between the optimal fluctuation potential $U(\mathbf{x})$ and the wave function $\psi(\mathbf{x})$ :

$$
U(\mathbf{x})=\lambda \int K\left(\mathbf{x}, \mathbf{x}^{\prime}\right)\left|\psi\left(\mathbf{x}^{\prime}\right)\right|^{2} d \mathbf{x}^{\prime},
$$

analogs to Eq. (16) for the uncorrelated disorder. This equation shows that the characteristic size of $U(\mathbf{x})$ is always equal to $b$. Indeed, it is correct if the characteristic size of the wave function is less or equal to $b$. In the opposite case the characteristic size of the potential would be much larger than $b$ and, respectively, the probability of such configuration would be much smaller. Deep levels have negative energy $E$, much larger by modulus than the energy scale of the random potential $U_{0}$. Since the optimal fluctuation potential well must have the level $E$, its depth is not less than $|E|$. The fundamental difference between the optimal potential wells in the cases of uncorrelated and strongly correlated disorder is that the former contains only one level, whereas the latter contains many levels as shown in Fig. 3. Indeed, the number of levels in the optimal well is not less than $1 / 6 \pi^{2}(\sqrt{2 m|E|} b / \hbar)^{3} \geq 1 / 6 \pi^{2}\left(\sqrt{2 m U_{0}} b / \hbar\right)^{3} \sim(b / \mathcal{L})^{3 / 4}$ $=(b / B)^{3} \gg 1$. Therefore, the level $E$ is located close to the bottom of the optimal potential well. Its depth with high precision is equal to $|E|$ and its shape is determined by the function $h(r / b)$ from Eq. (8). If the correlation function is nonsingular, then $U(\mathbf{x})=E h(r / b)$. The case when the correlation function is singular will be discussed separately at the end of the section.

The exponent in Eq. (1) can be calculated exactly leading to the result for the probability $q(E)$ to find the level with energy less than $E$ (with precision of a prefactor): 


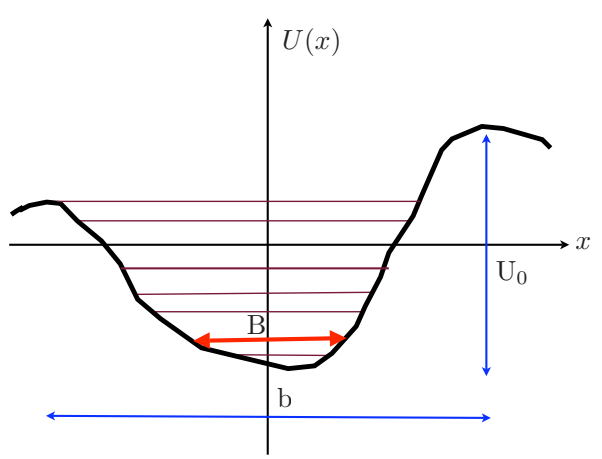

FIG. 3. (Color online) Long-range correlated potential. There are typically many bound states per well.

$$
q(E)=\exp \left(-\frac{E^{2}}{2\left\langle U^{2}\right\rangle}\right) .
$$

In a more accurate treatment one needs to take in account that the level $E$ can be not the ground state in the optimal well, but an excited state. Since even excited states are close to the bottom, the spectrum is the same as for a spherically symmetric quantum oscillator with the frequency $\omega$ $=\left[|E| h^{\prime \prime}(0) / m b^{2}\right]^{1 / 2}$. Therefore, the depth $V$ of the well is not exactly equal to $|E|$. It depends on what excited state occupies the particle:

$$
V_{n_{1}, n_{2}, n_{3}}=|E|+\left[\left(n_{1}+n_{2}+n_{3}\right)+3 / 2\right] \hbar \omega .
$$

After summation over all $n_{i}(i=1,2,3)$ from 0 to infinity we find the corrected expression for the probability $q(E)$ :

$$
q(E)=\exp \left(-\frac{E^{2}+3|E| \hbar \omega}{2\left\langle U^{2}\right\rangle}\right)\left[1-\exp \left(-\frac{|E| \hbar \omega}{\left\langle U^{2}\right\rangle}\right)\right]^{-3} .
$$

The ratio $|E| \hbar \omega /\left\langle U^{2}\right\rangle$ which appears in Eq. (49) by the order of magnitude is equal to the product of two ratios $\left(|E| / U_{0}\right)^{3 / 2} \times(B / b)$. The first factor is large; the second one is small and their product varies from small to large values always remaining larger than $(B / b)$. Nevertheless, a strong enhancement of probability up to a factor $(b / B)^{3}$ in comparison to the initial expression (47) is achieved at decreasing modulus of energy. Summarizing, we conclude that the possibility of the excited states may change a comparatively slow-varying prefactor leaving principal exponent (18) unchanged.

The density of potential wells containing deep levels lower than $E$ for strongly correlated random potential reads

$$
n_{w}(E, b)=b^{-3} \exp \left(-E^{2} / 2 U_{0}^{2}\right)
$$

and the average distance between such wells is

$$
d(E, b)=b \exp \left(E^{2} / 6 U_{0}^{2}\right) .
$$

The direct tunneling between wells does not play an important role since the particles remain almost classical even near the mobility edge in contrast to the uncorrelated disorder. The percolation of the classical trajectory corresponds to the mobility edge. According to the Shklovsky estimate, ${ }^{48}$ the percolation of wells happens at $E=-0.9 U_{0}$. The characteris- tic de Broglie wavelength $\hbar / \sqrt{2 m|E|}$ at this energy is much less than $b$ for strongly correlated disorder. The mobility edge energy is negative and not far from the percolation threshold.

If the correlation function is singular, as for the OZ correlator (6), the result of Eq. (47) is not valid. In this case the shape of the optimal fluctuation potential reads

$$
U_{0}(r)=-\alpha \frac{e^{-r / b}}{r},
$$

where the coefficient $\alpha$ must be found from the condition that a stationary state of this potential is equal to a fixed negative $E$. As in the previous case, let us first consider the case when $E$ is the ground-state energy of the OZ potential. We anticipate that the linear size of this state is much less than $b$. Then in the OZ potential (52) it is possible to put exponential factor equal to 1 . Thus, the problem is reduced to the quantum Kepler problem, whose spectrum and eigenstates are known. In particular the energy of the ground state reads

$$
E_{1}=-\frac{m \alpha^{2}}{2 \hbar^{2}}
$$

From the above indicated constraint we find the relation between $\alpha$ and $E$ :

$$
\alpha=\hbar \sqrt{\frac{2|E|}{m}} .
$$

The diameter of the ground state is equal to the Bohr radius:

$$
a_{B}=\frac{\hbar^{2}}{m \alpha}=\frac{\hbar}{\sqrt{2 m|E|}} .
$$

The inequalities $|E| \gg \kappa / b^{3 / 2}$ and $b \gg \mathcal{L}=\hbar^{4} / m^{2} \kappa^{2}$ must be satisfied. From these inequalities and Eq. (55) we find

$$
a_{B} \ll \frac{\hbar b^{3 / 4}}{\sqrt{m \kappa}}=\left(\frac{\mathcal{L}}{b}\right)^{1 / 4} b \ll b .
$$

This inequality justifies the earlier accepted approximation (neglect of the exponential factor). Now we are in a position to calculate the probability of the optimal state. The number in exponent reads

$$
\Phi=\frac{1}{2 \kappa^{2}} \int\left[U_{0}^{2}(r)+\kappa^{2}\left(\frac{d U_{0}}{d r}\right)^{2}\right] d \Omega .
$$

The two terms in the integral give identical contributions. Thus,

$$
\Phi=\frac{4 \pi \alpha^{2}}{\kappa^{2}} \int_{0}^{\infty} e^{-2 r / b} d r=\frac{4 \pi \hbar^{2} b|E|}{m \kappa^{2}} .
$$

Therefore, in this approximation the singular character of the OZ correlator leads to a probability

$$
q(E)=\exp \left[-\frac{4 \pi \hbar^{2} b|E|}{m \kappa^{2}}\right],
$$

which is not the Gaussian. 
Let now assume that the energy $E$ corresponds to one of the excited states:

$$
|E|=\frac{m \alpha^{2}}{2 \hbar^{2} n^{2}} .
$$

From this equation we find the value $\alpha_{n}$ corresponding to the $n$th excited state:

$$
\alpha_{n}=n \alpha .
$$

Therefore, from Eq. (58) the corresponding value of the number in the exponent is $\Phi_{n}=n^{2} \Phi$. Since $\Phi \gg 1$, the series $\Sigma \exp \left(-\Phi_{n}\right)$ with high precision is equal to its first term.

\section{B. Weakly repulsive Bose gas in strongly correlated disordered potential}

In this section we consider the Bose gas with the average density $n$. The gas partly fills all potential wells with the depth less than $E$. Let the particles fill a typical well up to the radius $R<b$. If the correlator is regular at $\mathbf{x}=\mathbf{x}^{\prime}$, the following equation gives a rough estimate of energy per particle:

$$
\mu(E, R ; b, n)=E\left(1-\frac{R^{2}}{2 b^{2}}\right)+\frac{3 \hbar^{2} n a}{m}\left(\frac{b}{R}\right)^{3} \exp \left(\frac{E^{2}}{2 U_{0}^{2}}\right) .
$$

The first term in the right-hand side of Eq. (62) is the gain of energy due to the random potential; the quadratic term in the brackets corresponds to the oscillatorlike potential at small $R$; the second term is the energy of interaction. Minimizing $\mu(E, b, R)$ over $E$ and the ratio $R / b$, one arrives at following results:

$$
\begin{gathered}
E \approx-U_{0} \sqrt{2 \ln \frac{n_{c}}{n}}, \\
\frac{R}{b} \approx\left(\ln \frac{n_{c}}{n}\right)^{-1 / 2} .
\end{gathered}
$$

The critical density $n_{c}$ is defined as follows:

$$
n_{c} \approx \frac{\sqrt{3}}{4} \frac{m U_{0}}{\hbar^{2} a} \sim \frac{1}{B^{2} a} .
$$

As in the case of the uncorrelated disorder it can be treated as a density at which the interaction energy $n g$ becomes equal to the attractive energy of the random potential $\sim U_{0}$. All previous results are valid at $n \ll n_{c}$. According to Eq. (64), the ratio $\frac{R}{b}$ is small. This fact justifies the approximation of quadratic potential in Eq. (62). It allows to estimate the characteristic momentum of particles or the width of their distribution over momentum:

$$
\Delta p=m \omega R=m \omega b\left(\ln \frac{n_{c}}{n}\right)^{-1 / 2} .
$$

The chemical potential is readily derived from Eqs. (62)-(65):

$$
\mu(b, n)=-U_{0} \sqrt{2 \ln \frac{n_{c}}{n}}\left[1-\frac{9}{4}\left(\ln \frac{n_{c}}{n}\right)^{-1}\right] .
$$

The compressibility is

$$
\frac{\partial n}{\partial \mu}=\frac{n \sqrt{2 \ln \frac{n_{c}}{n}}}{U_{0}}\left(1-\frac{9}{4 \ln \frac{n_{c}}{n}}\right) .
$$

Two filled wells are separated by a typical distance $d(b, n)$ much larger than the size of the well:

$$
d(b, n)=b\left(\frac{n_{c}}{n}\right)^{1 / 3} .
$$

With this value of $d$ it is ready to estimate the tunneling coefficient:

$$
t(b, n) \approx \exp \left[-\frac{b}{B}\left(\frac{n_{c}}{n}\right)^{1 / 3}\right] .
$$

The number of particles in each typical well can be estimated as $\mathcal{N}=n / n_{w}(E)=n_{c} b^{3} \approx b^{3} / B^{2} a$. It is large if $b \geq a$.

These results conclude the description of localized states for strongly correlated disorder. At energies between $-U_{0}$ and $U_{0}$ the motion of particle is strongly disordered, but it becomes more and more free when energy approaches and exceeds $U_{0}$. At $E \gg U_{0}$, the random potential can be considered as a perturbation. The quantum phase transition from the localized random singlet state to a superfluid proceeds at $n=n_{c}$. Note that the critical density in the presence of strongly correlated disorder does not depend on the correlation length $b$.

\section{BOSE GAS IN A TRAP WITH DISORDERED POTENTIAL}

Here we consider deeply localized states in a potential which is the sum of a harmonic trap and a Gaussian random potential. The Hamiltonian of the system has an additional term namely the potential energy of Bose particles in the trap:

$$
H_{\text {trap }}=\int V_{\text {trap }}(\mathbf{x}) \psi^{\dagger}(\mathbf{x}) \psi(\mathbf{x}) d \mathbf{x},
$$

where the harmonic potential of the trap generally has a form

$$
V_{\text {trap }}(\mathbf{x})=\frac{m}{2}\left(\omega_{x}^{2} x^{2}+\omega_{y}^{2} y^{2}+\omega_{z}^{2} z^{2}\right) .
$$

In this section we consider only the isotropic trap with $\omega_{x}$ $=\omega_{y}=\omega_{z}=\omega$. Strongly anisotropic traps can be considered as a system with reduced dimension 2 or 1 and will be considered in the next section. A new scale of length associated with the trap is the well-known oscillator length $\ell$ $=\sqrt{\hbar /(m \omega)}$.

A principal difference between the Bose gas in a box and in a trap is that in the latter case the gas forms a cloud whose size is determined by energy minimization at a fixed number 
of particles, whereas in the former case the size of the box and, therefore, its average density $n$ is fixed. Nevertheless, we will see that all phase states of the Bose gas in the box appear when this gas is placed into a harmonic trap, but their appearance is regulated by the total number of particles in the gas $N$ instead of their average density.

\section{A. Trap with uncorrelated disorder}

Four competing parts of a Bose-particle energy are kinetic energy, the confining potential energy of the trap, the repulsion from other particles, and the energy of the random potential. Two of them, the interaction with the trap and the random potential, tend to confine and localize the particle. Let the gas form a single cloud of the radius $R$. The rough estimate of the two confining energy contributions can be done as follows: ${ }^{47}$

$$
V_{\text {trap }}=\frac{m \omega^{2} R^{2}}{2}=\frac{\hbar^{2}}{2 m} \frac{R^{2}}{\ell^{4}} .
$$

The disorder energy for the same cloud can be estimated as

$$
V_{\mathrm{dis}}=-\frac{\kappa}{\left(\frac{4 \pi R^{3}}{3}\right)^{1 / 2}} \approx-\frac{\hbar^{2}}{2 m}\left(\frac{\mathcal{L}}{R}\right)^{3 / 2} .
$$

The latter estimate can be justified as follows. The cloud chooses such a position that the random potential energy is negative. A characteristic fluctuation of energy in a volume $\Omega$ is

$$
\begin{aligned}
\frac{1}{\Omega}\left\langle\left[\int_{\Omega} U(\mathbf{x}) d \mathbf{x}\right]^{2}\right\rangle^{1 / 2} & =\frac{1}{\Omega}\left[\iint_{\Omega} K\left(\mathbf{x}, \mathbf{x}^{\prime}\right) d \mathbf{x} d \mathbf{x}^{\prime}\right]^{1 / 2} \\
& =\frac{\kappa}{\sqrt{\Omega}} .
\end{aligned}
$$

Substituting into the last expression $\Omega=\frac{4}{3} \pi R^{3}$, we arrive at Eq. (73). Comparing the two confining contributions, we conclude that, at $\mathcal{L} \gg \ell$, the influence of disorder is negligible. The remaining two terms of energy: kinetic energy

$$
K \sim \hbar^{2} /\left(2 m R^{2}\right)
$$

and the repulsion energy per particle:

$$
V_{\text {int }}=g n=\frac{\hbar^{2}}{m} \frac{3 N a}{R^{3}}
$$

are deconfining. The kinetic energy dominates at $R \gg 3 \mathrm{Na}$. In the opposite case $R \ll 3 N a$ the repulsion energy dominates. Below we analyze several limiting cases.

(1) Weak disorder: $\mathcal{L} \gg \ell$. Depending on the relative value of the interaction one should distinguish two different situations as follows:

(1a) Weak interaction: $3 N a \ll \ell$. In this case the interaction can be neglected. Minimizing two remaining terms, the kinetic energy and energy of the trap, we find $R=\ell$. Physically it means that all particles are condensed at the oscillator ground state.

(1b) Strong interaction: $3 N a \gg \ell$. Neglecting kinetic en-

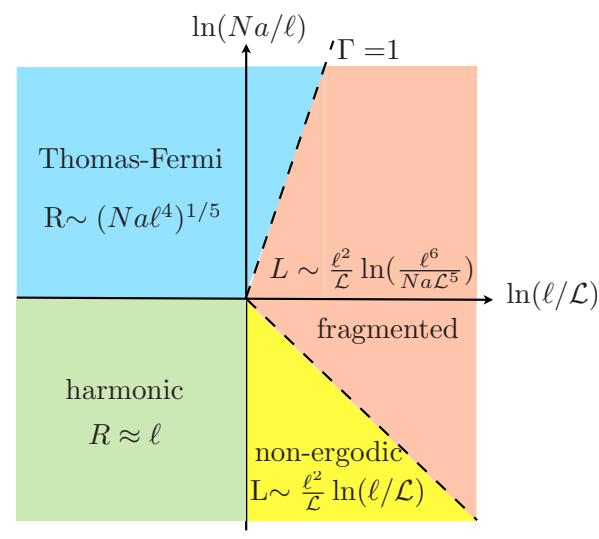

FIG. 4. (Color online) Regime diagram of atoms in threedimensional traps: uncorrelated disorder. $R$ denotes the size of the single existing atomic cloud. $L$ is the size of the cloud of fragments.

ergy and minimizing remaining energy of traps plus the repulsion energy, one finds the result known as Thomas-Fermi approximation: ${ }^{70} R=\left(\frac{9}{2} \mathrm{Na} \ell^{4}\right)^{1 / 5}$.

More interesting is the case of strong disorder:

(2) Strong disorder: $\mathcal{L} \ll \ell$. Again we consider several different limiting cases depending on relative strength of disorder, interaction, and the trap potential.

(2a) Weak interaction: $3 N a \ll \mathcal{L} \ll \ell$. In this range of variables the nonergodic phase is realized. Since interaction is negligible, the particles find a random potential well with the deepest level and fall into it. Let such a well can be found at a distance $\sim L$ from the trap center. Its depth typically is about $9 \mathcal{E} \ln ^{2}(L / \mathcal{L})$. This gain of energy must be not less than the loss of the trap energy $m \omega^{2} L^{2} / 2$. A typical value of $L$ appears when both these energies have the same order of magnitude. Then $L \approx 6 \sqrt{2}\left(\ell^{2} / \mathcal{L}\right) \ln (\ell / \mathcal{L})$. A typical size of the well is $R \approx \mathcal{L} /[6 \ln (\ell / \mathcal{L})]$.

(2b) Moderate interaction: $\mathcal{L} \ll 3 N a \ll \ell$. In this case the ergodicity is restored. Our experience with the gas in a box prompts that the gas cloud is split into fragments each occupying a random potential well from very small size until some size $R$ depending on $N$. From Eq. (29) the typical disorder energy per particle is $\mu=-\mathcal{E}\left(\ln \frac{n_{c}}{n}\right)^{2}$. It becomes equal to the trap energy at a distance $L \sim\left(\ell^{2} / \mathcal{L}\right) \ln \Gamma$ [see the definition of the parameter $\Gamma$ below in Eq. (76)]. Therefore, the average density is $n \sim N \mathcal{L}^{3} / \ell^{6}$. The ratio $n_{c} / n$ plays the decisive role: the state of the Bose gas is fragmented and strongly localized when it is large; the transition to delocalized superfluid state proceeds when this ratio becomes 1 . With precision of a numerical factor the ratio $n_{c} / n$ is equal to a new dimensional parameter:

$$
\Gamma=\frac{\ell^{6}}{3 N a \mathcal{L}^{5}} .
$$

Strongly localized fragmented state is realized at $\Gamma \gg 1$. The transition proceeds at $\Gamma=1$. The superfluid phase emerges at $\Gamma<1$. The regime diagram is shown in Fig. $4 .^{71}$ Note the counterintuitive dependence of the cloud size on the number of particles: the cloud slightly contracts with increasing number of particles. It happens because the number of particles in 


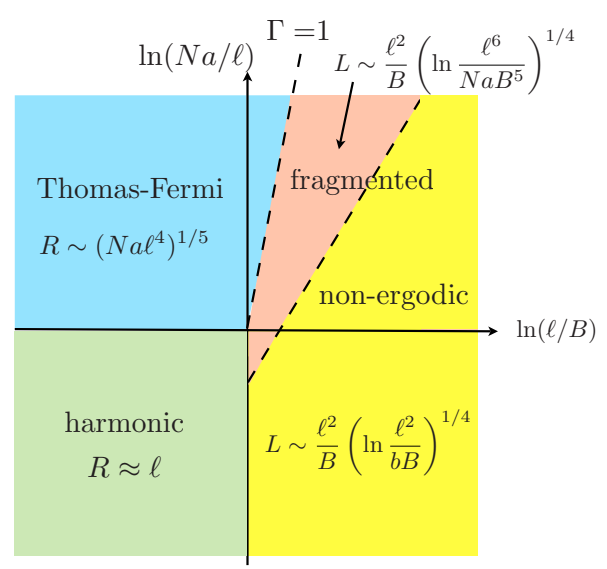

FIG. 5. (Color online) Regime diagram of atoms in threedimensional traps: correlated disorder. $R$ denotes the size of the single atomic cloud; $L$ denotes the size of the fragmented state.

each fragment increases more rapidly with the average density than the number of fragments.

\section{B. Trap with strongly correlated disorder}

Following the line of development accepted in the previous subsection, we start with the case of weak disorder:

(1) Weak disorder: $U_{0} \ll \hbar \omega$ or $B \gg \ell$. In this case the random potential only slightly changes the state of the gas in the absence of disorder (see previous subsection). In what follows we assume for simplicity that $\ell \geq b$.

(2) Strong disorder: $U_{0} \gg \hbar \omega$ or $B \ll \ell$. As in the previous subsection we consider different relative strength of the interaction and disorder.

(2a) Weak interaction: $3 N a \ll b^{3} / B^{2} \ll \ell$. The interaction is negligible. Therefore, all particles find the deepest well in the trap and fall into it. The typical distance from the center of the trap to the deepest well is $L=\left(\ell^{2} / B\right)\left(\ln \ell^{2} / b B\right)^{1 / 4}$ and its typical depth is $E=-U_{0} \sqrt{6 \ln \frac{L}{b}}$. In this range of parameters the system is nonergodic.

(2b) Moderate interaction: $b^{3} / B^{2} \ll 3 N a \ll \ell$. In this case the particles are distributed among multiple potential wells. The system is ergodic. The average depth of the well is $E(N)=-U_{0} \sqrt{2 \ln \Gamma}$, where

$$
\Gamma=\frac{U_{0}^{5 / 2}}{\hbar^{2} N^{1 / 2} \omega^{3}}=\frac{\ell^{6}}{N a B^{5}}
$$

is the dimensionless parameter analogs to that introduced in the previous subsection. It represents the ratio $n_{c} / n$. Until $\Gamma$ is large the ground state of the Bose gas is deeply localized random singlet. The transition to the superfluid state proceeds at $\Gamma \sim 1$. The total linear size of the cloud is $L$ $=2^{3 / 4}\left(\ell^{2} / B\right)(\ln \Gamma)^{1 / 4}$. Inside a fluctuation potential which has a typical size $b$ particles occupy a smaller sphere of the radius $R \sim\left(N a b^{2} B^{2}\right)^{1 / 5}$. The phase state (regime) of the system depends on three dimensionless parameters: $B / b, 3 \mathrm{Na} / \ell$, and $\ell / b$. The regime diagram is shown on Fig. 5 .

\section{LOWER DIMENSIONS}

In the experiments with cooled gases the trap is mostly realized as a rotation ellipsoid with a large aspect ratio. In the experimental setup with $\mathrm{He}$ on Vycor experimenters used thin helium film, which displayed the crossover from 2D to 3D behavior. Therefore, and also for comparison with exact $1 \mathrm{D}$ solution $^{33}$ it is reasonable to extend our theory for lower dimensions $d=1,2$.

\section{A. Box: Uncorrelated disorder}

\section{Single-particle levels}

We start with the extension of Eq. (11) connecting the Larkin length $\mathcal{L}_{d}$ and the constant $\kappa^{2}$ for the uncorrelated disorder:

$$
\mathcal{L}_{d}=\left(\frac{\hbar^{4}}{m^{2} \kappa^{2}}\right)^{1 /(4-d)}
$$

following from the dimension consideration. The characteristic energy reads

$$
\mathcal{E}_{d}=\frac{\hbar^{2}}{2 m \mathcal{L}_{d}^{2}}=\frac{1}{2}\left(\frac{m^{d} \kappa^{4}}{\hbar^{2 d}}\right)^{1 /(4-d)} .
$$

The extension of exponential law (17) for the probability to find the deep potential well of the size not larger than $R$ is

$$
q(R)=f \exp \left[-\left(\frac{\mathcal{L}_{d}}{R}\right)^{4-d}\right]=f \exp \left[-\left(\frac{|E|}{\mathcal{E}_{d}}\right)^{(4-d) / 2}\right],
$$

where the Larkin length differs from that given by definition (78) by the factor $\left(\Omega_{d} / 2\right)^{1 /(4-d)}$, in which $\Omega_{d}$ $=2^{d-1} \pi^{d / 2}(d !)^{-1} \prod_{k=1}^{d-2} \frac{\Gamma[(k+1) / 2]}{\Gamma[(k+2) / 2]}$ is the volume of the sphere with the radius 1 in $d$-dimensional space. As earlier $f$ is a powerlike function of the ratio $\mathcal{L} / R$ at $\mathcal{L} / R \gg 1: f(x) \propto x^{\alpha}$ with $\alpha$ $=1$ for $d=2,3$ and $\alpha=0$ for $d=1 .^{51}$ The extension of Eq. (19) for the density of the fluctuation potential wells with the radius not exceeding $R$ looks in $d$ dimensions as follows:

$$
n_{w}(R)=R^{-d} f \exp \left[-\left(\frac{\mathcal{L}_{d}}{R}\right)^{4-d}\right]
$$

and the average distance between such wells is

$$
d(R)=R f^{-1 / d} \exp \left[\frac{1}{d}\left(\frac{\mathcal{L}_{d}}{R}\right)^{4-d}\right] .
$$

The tunneling amplitude between nearest wells has a characteristic value

$$
t(R)=\exp \left\{-f^{-1 / d} \exp \left[\frac{1}{d}\left(\frac{\mathcal{L}_{d}}{R}\right)^{4-d}\right]\right\} .
$$

\section{2. d-dimensional gas in a box}

We consider the filling of the potential wells by the diluted Bose gas of the density $n=N / L^{d}$. Again, let the gas fill all the wells up to the radius $R$. The average number of particles per well is 


$$
\mathcal{N}(R)=\frac{n}{n_{w}(R)}=f^{-1} n R^{d} \exp \left[\left(\frac{\mathcal{L}_{d}}{R}\right)^{4-d}\right]
$$

and the density of particles in the well is

$$
n_{p}(R)=\mathcal{N}(R) /\left(\Omega_{d} R^{d}\right)=\frac{n}{f \Omega_{d}} \exp \left[\left(\frac{\mathcal{L}_{d}}{R}\right)^{4-d}\right] .
$$

The repulsion energy per particle is as before $g_{d} n_{p}(R)$, but the connection between the coupling constant $g$ and the scattering amplitude $a$ depends on dimensionality of the space:

$$
g_{d}=\frac{\Omega_{d} \hbar^{2} a^{d-2}}{m} .
$$

At $d=2$ this equation is invalid, but the coupling constant $g$ is well defined and has dimensionality energy $\times$ length $^{2}$. Minimizing the energy per particle in complete analogy with what was done in Sec. IV, we find the maximal radius of the filled well as a function of density,

$$
R(n)=\frac{\mathcal{L}_{d}}{\left(\ln \frac{n_{c}}{n}\right)^{1 /(4-d)}},
$$

and the critical density is defined by the equation

$$
n_{c}=\frac{\Omega_{d} \mathcal{E}_{d}}{g_{d}(4-d)}=\frac{\Omega_{d}}{4 \pi \mathcal{L}_{d}^{2} a^{d-2}(4-d)} .
$$

In 1D Bose gas the critical density increases with the scattering amplitude in contrast to two- and three-dimensional cases. The reason is that the 1D Bose gas with strong repulsion (hard core) is equivalent to the Fermi gas whose kinetic energy increases with the size of the core.

The ratio $n_{c} / n$ can be rewritten in equivalent form:

$$
\frac{n_{c}}{n}=\left(\frac{\xi_{d}}{\mathcal{L}_{d}}\right)^{2},
$$

where $\xi_{d}=\hbar / \sqrt{2 m g n}$ is the coherence or healing length of the Bose gas without disorder. The condition $n \ll n_{c}$ at which the deeply localized ground state is realized implies also $\mathcal{L}_{d}$ $\ll \xi_{d}$ as it could be expected. In 1D case large values of the gas parameter $n a_{1}$ correspond to the almost ideal case in which kinetic energy is much larger than the interaction. Accordingly, the gas parameter inside the fluctuation well $n_{c} a^{d} \sim\left(a / \mathcal{L}_{d}\right)^{2}$ must be large in 1D case.

We do not show other calculations analogous to those produced in Sec. IV and present only the final results as follows: number of particles in a filled well,

$$
\mathcal{N}(n)=\Omega_{d}^{-1}\left(\ln \frac{n_{c}}{n}\right)^{-d /(4-d)}\left(\frac{\mathcal{L}_{d}}{a}\right)^{d-2},
$$

distance between the filled wells:

$$
d(n)=\mathcal{L}_{d}\left(\frac{n_{c}}{f n}\right)^{1 / d}\left(\ln \frac{n_{c}}{n}\right)^{-1 /(4-d)},
$$

chemical potential:

$$
\mu(n)=-\mathcal{E}_{d}\left(\ln \frac{n_{c}}{n}\right)^{2 /(4-d)},
$$

tunneling amplitude between wells:

$$
t(n)=\exp \left[-\left(f \frac{n_{c}}{n}\right)^{1 / d}\right] .
$$

\section{Critical phenomena in d-dimensional gas}

From Eq. (88) it follows that the quantum phase transition proceeds on the line

$$
n_{c} \approx \frac{1}{a^{d-2} \mathcal{L}_{d}^{2}}, \text { i.e., } \xi_{d} \approx \mathcal{L}_{d},
$$

where $\mathcal{L}_{d}=\left(\hbar^{2} / m \kappa\right)^{2 /(4-d)}$ and $\xi_{d} \sim\left(a^{d-2} n\right)^{-1 / 2}$ denote the $d$-dimensional Larkin and healing length, respectively.

Important characteristics of the critical phenomena are the marginal space dimensions. For weak disorder, the Larkin length diverges at $d \rightarrow 4$. Hence, in dimensions $d>4$ disorder has to overcome a threshold value to destroy superfluidity even in the limit of weak interaction. Thus, $d=4$ is an upper critical dimension of the problem as has been discussed already in Ref. 31. A lower critical dimension is $d=1$. This can be seen from calculation of the ground-state energy Eq. (43) in one dimension which is logarithmically infrared divergent reflecting strong quantum fluctuation. Giamarchi and Schul $\mathrm{z}^{33}$ in a seminal paper found a quantum phase transition in 1D Bose gas in disordered environment from a superfluid to an insulating phase at increasing interaction parameter $K$ $\approx 2 / 3$ (compare the Appendix). Since then this transition was considered as a theoretical counterpart of the real transition in 1D systems (see, e.g., Ref. 31). However, at this transition $\xi_{1} n \approx 0.25$ strongly violating the gas condition $\xi_{1} n \gg 1$. Thus, we conclude that the transition found in Ref. 33 is irrelevant to the experimental transition in onedimensional gas clouds at $n \approx n_{c}$. Giamarchi and Schulz, however, claimed that there are two different phase transitions at small disorder: one at large value of dimensionless coupling constant $K$ and another at small $K$. Unfortunately the part of the phase diagram beyond a small vicinity of the critical point $K=2 / 3, \kappa=0$ was obtained by a speculation rather than a rigorous treatment. Therefore, the question about the existence of two different quantum phase transitions remains open.

Critical behavior near the quantum transition was studied by Halperin et $a .^{30}$ and by Fisher et $a .^{31}$ In the latter work the scaling consideration was developed and even the critical exponents were found. However, we are not sure that they are valid for our problem since, according to previous remark, they are associated with the strong-coupling critical point. Second, even if there is only one critical point, the marginal dimensions are $1+1$ and $4+1$ and it is very doubtful that exact critical exponent can be found in the case 3 +1 (see a recent review ${ }^{34}$ ).

\section{Strongly anisotropic traps as low-dimensional systems}

Considering the gas in a trap which has a shape of a disk or a cigar, one should express the parameters of effective 
$d$-dimensional problem in terms of parameters of the 3D gas: the particle density $n, 3 \mathrm{D}$-scattering length $a$, Larkin length $\mathcal{L}$, the transverse oscillator length $\ell_{\perp}$, and the longitudinal oscillator length $\ell$. To simplify the results we consider only the most interesting and experimentally accessible situation of a weak confinement:

$$
a \ll \ell_{\perp} \ll \mathcal{L} .
$$

Then it is straightforward to see that the effective $d$-dimensional Larkin length $\mathcal{L}_{d}$ is related to the 3D Larkin length $\mathcal{L}$ by the following relationship: ${ }^{47}$

$$
\mathcal{L}_{d} \approx\left(\mathcal{L} \ell_{\perp}^{3-d}\right)^{1 /(4-d)} .
$$

Indeed, the argument in exponent of the Gaussian distribution for uncorrelated disorder is $\sim U^{2} \Omega / 2 \kappa^{2}$. The volume $\Omega$ can be approximately factorized: $\Omega \simeq \Omega_{d} R^{d} \Omega_{3-d} \ell_{\perp}^{3-d}$. Using the standard estimate for $U=-\hbar^{2} / m R^{2}$, we arrive at Eq. (80) with $\mathcal{L}_{d}$ defined by Eq. (96) with precision of a numerical factor. To find the effective scattering length $a_{d}$, one should use an obvious identity $n g=n_{d} g_{d}$ (both expressions are the interaction energy per particle) with $n_{d}=n \ell_{\perp}^{3-d} \Omega_{3-d}$ being the density in the reduced dimensionality space. From these two equations it follows that

$$
g_{d}=\frac{g \ell_{\perp}^{d-3}}{\Omega_{3-d}} .
$$

Employing Eqs. (86) and (23), we arrive at following relationship:

$$
a_{d} \sim\left(a \ell_{\perp}^{d-3}\right)^{1 /(d-2)} .
$$

This equation is invalid for $d=2$ and instead Eq. (97) must be used. Below we consider possible regimes similarly to what we did for the 3D system.

(1) Weak disorder: $\mathcal{L}_{d} \gg \ell$ or equivalently $\mathcal{L} \gg \ell^{4-d} / \ell_{\perp}^{3-d}$. The random potential is negligible. The competition of the kinetic energy, trap energy, and interaction provides two different regimes as follows:

(a) Weak interaction: $g_{d} N \ll \hbar^{2} \ell^{d-2} / m$ or equivalently $N a$ $\ll \ell^{d-2} \ell_{\perp}^{3-d}$. In this case the longitudinal size of the cloud $R$ coincides with $\ell$.

(b) Strong interaction: $N a \gg \ell^{d-2} \ell_{\perp}^{3-d}$. This is the region of the Thomas-Fermi regime $R \sim\left(\mathrm{Na}^{4} / \ell_{\perp}^{3-d}\right)^{1 /(d+2)}$.

(2) Strong disorder: $\mathcal{L}_{d} \ll \ell$ or equivalently $\mathcal{L} \ll \ell^{4-d} / \ell_{\perp}^{3-d}$.

(a) Weak interaction: $g N / \mathcal{L}_{d}^{d} \ell_{\perp}^{3-d} \ll \hbar^{2} / m \mathcal{L}_{d}^{2}$ or equivalently $N a \ll \mathcal{L}^{(d-2) /(4-d)} \ell_{\perp}^{2(3-d) /(4-d)}$. This is a nonergodic situation: all particles fall into the deepest potential well of the typical size $R \sim \mathcal{L}_{d} /\left(\ln \frac{\ell}{\mathcal{L}_{d}}\right)^{1 /(4-d)}$. For $d=2$ it happens at $N \ll \ell_{\perp} / a$; for $d=1$ the nonergodic regime exists if $\ell_{\perp}$ $\gg\left(a^{3} \mathcal{L}\right)^{1 / 4}$.

(b) Moderate interaction: $\quad \mathcal{L}^{(d-2) /(4-d)} \ell_{\perp}^{2(3-d) /(4-d)} \ll N a$ $\ll \ell^{d-2} \ell_{\perp}^{3-d}$. In this range of interaction the cloud consists of multiple fragments. They are weakly coupled each to other provided the dimensionless parameter $\Gamma=\ell^{2 d} /\left(N a_{d}^{d-2} \mathcal{L}_{d}^{d+2}\right)$ $=\ell_{\perp}^{2 d} /\left(N a \mathcal{L}^{(d+2) /(4-d)} \ell_{\perp}^{[2(3-d)(d-1) /(4-d)]}\right)$ is large (note that the exponent at $\ell_{\perp}$ is zero at $d=1,3$ and -1 at $\left.d=2\right)$. We remind that $\Gamma$ is equivalent to the ratio $n_{c} / n$. A typical longitudinal size of the fragment is $R=\mathcal{L}_{d} /(\ln \Gamma)^{1 /(4-d)}$; a typical distance between fragments is $d=R \Gamma^{1 / d}$; the tunneling amplitude be-

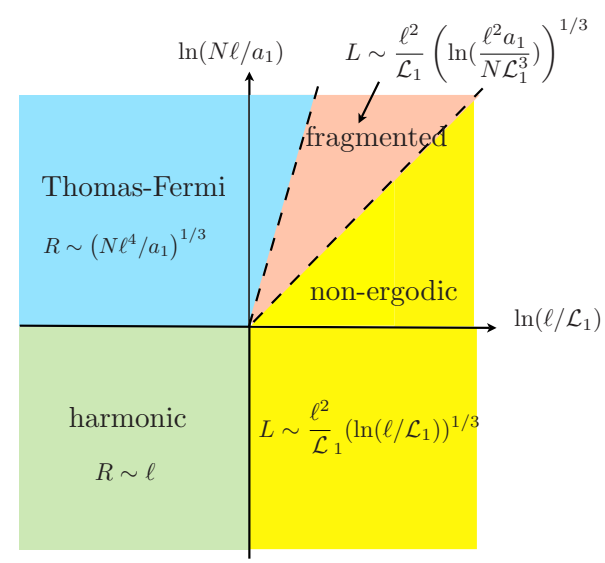

FIG. 6. (Color online) Regime diagram of atoms in a onedimensional trap: uncorrelated disorder. In reduced dimensions $R$ denotes the longitudinal size of the single atomic cloud; $L$ denotes the longitudinal size of the fragmented state.

tween fragments is $t \sim \exp \left(-\Gamma^{1 / d}\right)$. The transition from localized state to the phase-correlated superfluid state proceeds at $\Gamma \approx 1$. At $\Gamma \gg 1$ the disorder is negligible and the ThomasFermi approximations works. The phase diagram in one dimension is shown in Fig. 6.

\section{B. Strongly correlated disorder}

As in $3 \mathrm{D}$ case, the distribution of random potential is characterized by the correlation length $b$ and the characteristic energy $U_{0}$. It is assumed that $b \gg \mathcal{L}$ $\sim\left[\hbar^{4} /\left(m^{2} U_{0}^{2} b^{d}\right)\right]^{1 /(4-d)}$ or equivalently $b \gg B=\hbar / \sqrt{m U_{0}}$. The characteristic size of a deep potential well is equal to the correlation length $b$. The probability to find the well containing the level with energy $E<0$ and sufficiently deep, so that $|E| \gg U_{0}$, is given by the Gaussian exponent Eq. (47). The number of such wells per unit volume is

$$
n_{w d}(E)=b^{-d} \exp \left(-\frac{E^{2}}{2 U_{0}^{2}}\right)
$$

and the distance between them is $d_{d}(E)=\left[n_{w d}(E)\right]^{-1 / d}$. The gas with the average density $n_{d}$ fills the wells up to the depth $|E|=U_{0} \sqrt{\ln \left(n_{\mathrm{cd}} / n_{d}\right)}$. The critical density is given by equation

$$
n_{\mathrm{cd}}=\frac{\Omega_{d}}{d} \frac{U_{0}}{g_{d}}=\frac{\Omega_{d}}{4 \pi d} \frac{1}{B^{2} a_{d}^{d-2}} .
$$

The latter formula is invalid at $d=2$, but the former one, employing the value $g_{d}$, remains correct. The particles in each well fill a $d$-dimensional sphere of the radius $R$ $=b U_{0} /|E|=b / \sqrt{\ln \left(n_{\mathrm{cd}} / n_{d}\right)}$. The transition to the superfluid state proceeds at $n_{d}=n_{\text {cd }}$.

Proceeding to the gas in a trap, we first specify conditions which are realistic and allow to reduce the number of possible regimes:

$$
a \ll \ell_{\perp} \ll \mathcal{L} \ll b .
$$

At these conditions $g_{d} \ell_{\perp}^{3-d}=g ; a_{d}^{d-2} \ell_{\perp}^{3-d}=a$ and Eq. (100) can be rewritten as follows: 


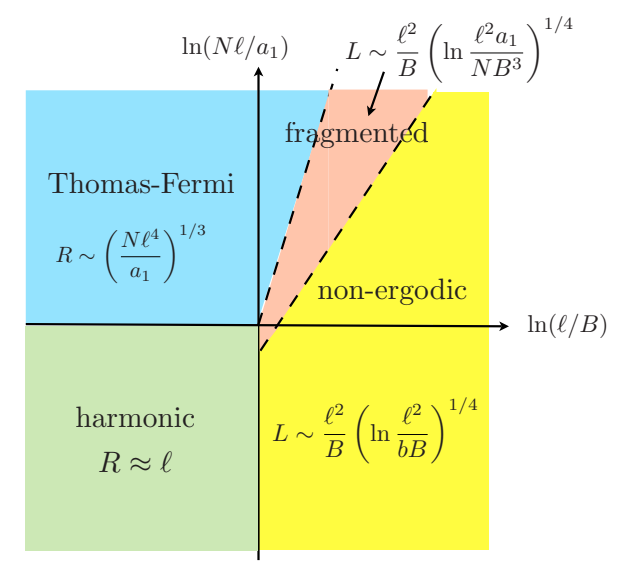

FIG. 7. (Color online) Regime diagram of atoms in a onedimensional trap: correlated disorder. In reduced dimensions $R$ denotes the longitudinal size of the single atomic cloud; $L$ denotes the longitudinal size of the fragmented state.

$$
n_{\mathrm{cd}}=\frac{\Omega_{d}}{d} \frac{U_{0} \ell_{\perp}^{3-d}}{g}=\frac{\Omega_{d}}{4 \pi d} \frac{\ell_{\perp}^{3-d}}{B^{2} a} .
$$

Note that this equation is valid at $d=2$ as well. The possible regimes are classified as follows:

(1) Weak disorder: $U_{0} \ll \hbar \omega$ or equivalently $B \gg \ell$.

(1a) Weak interaction: $N a \ll \ell^{d-2} \ell_{\perp}^{3-d}$. In this case the size of the cloud $R=\ell$.

(1b) Strong interaction: $N a \gg \ell^{d-2} \ell_{\perp}^{3-d}$. The ThomasFermi range:

$$
R=\left(\frac{4 \pi d}{\Omega_{d} \Omega_{3-d}} \frac{N a \ell^{4}}{\ell_{\perp}^{3-d}}\right)^{1 /(d+2)} .
$$

(2) Strong disorder: $U_{0} \gg \hbar \omega$ or $B \ll \ell$.

(2a) Weak interaction: $g N / b^{d} \ell_{\perp}^{3-d} \ll U_{0}$ or equivalently $N a \ll b^{d} \ell_{\perp}^{3-d} / B^{2}$; nonergodic situation: a typical depth of the deepest potential well is $E=-U_{0} \sqrt{2 d \ln (L / b)}$. A typical distance of this well from the center of the trap is $L$ $\sim \sqrt{U_{0} /\left(m \omega^{2}\right)}\left(2 d \ln \ell^{2} / b B\right)^{1 / 4}=\ell^{2} / B\left(2 d \ln \ell^{2} / b B\right)^{1 / 4}$.

(2b) Intermediate interaction: $b^{d} \ell_{\perp}^{3-d} / B^{2} \ll N a \ll \ell^{d-2} \ell_{\perp}^{3-d}$. This is the range of fragmented state. The particles in fragments are deeply localized if the parameter $\Gamma$ $=\ell^{2 d} \ell_{\perp}^{3-d} /\left(B^{d+2} a N\right)$ is large. The particles in a fragment occupy a $d$-dimensional sphere of the radius $R(N)=b / \sqrt{\ln \Gamma}$. Their typical energy is $E=-U_{0} \sqrt{\ln \Gamma}$. A typical distance between fragments is $d(N)=b \Gamma^{1 / d}$. The number of particles inside each fragment is about $b^{d} \ell_{\perp}^{3-d} /\left(a B^{2}\right)$. The total size of the cloud is $\sim\left(\ell^{2} / B\right)(\ln \Gamma)^{1 / 4}$. The transition to the superfluid states proceeds at $\Gamma \approx 1$. The phase diagram in one dimension is shown in Fig. 7.

\section{DISCUSSION AND CONCLUSIONS}

The theory predicts many quantitative characteristics of deeply localized states. It also gives simple formulas for the critical density $n_{c}$ at which the quantum phase transition from the random singlet to superfluid state proceeds. Below we discuss how these predictions can be checked in experiments with cooled atomic gases. Four important parameters can be controllably and independently varied in the experiment. They are the number of particles $N$; the frequency $\omega$ or equivalently the strength of the trap; the scattering length $a$ (by approaching one of the Feshbach resonances); the strength of disorder $U_{0}$. Using this freedom, it is feasible to pass all spectrum of regimes described above. In particular, varying $U_{0}$ it is possible to transit from uncorrelated to strongly correlated disorder. A simple estimate shows that, at $b \sim 1 \mu \mathrm{m}$, the transition from uncorrelated to strongly correlated regime proceeds at frequency of disorder potential $\omega_{d}=\sqrt{2 U_{0} / m b^{2}} \sim 1 \mathrm{kHz}$ which is accessible.

Simplest experiments are the measurements of the cloud size $L$ as a function of different variable parameters in the regime of multiple localized fragments. Our theory predicts that in the regime of uncorrelated disorder the size of the cloud is inversely proportional to the frequency of the harmonic trap $\omega$ and proportional to the square of the disorder strength. It also predicts a weak dependence of the size on the number of particles $\sim \ln N$. In the case of strongly correlated disorder the size of the cloud is again inversely proportional to $\omega$, but the dependence on the disorder strength is much weaker: $L \propto U_{0}^{1 / 2}$. The dependence on $N$ also is weaker than in the uncorrelated regime: $L \propto(\ln N)^{1 / 4}$.

It would be important to observe a crossover from the nonergodic state with one or few fragments to the ergodic state with many fragments and check that it happens at $N$ $=\mathcal{L} / 3 a$ for uncorrelated disorder and at $N=b^{3} / 3 a B^{2}$ for strongly correlated disorder (see modifications of these results for $d=1,2$ in Sec. VII). Counting the number of fragments, one can find the number of particles per fragment and compare it with theoretical prediction. Direct measurements of the size of fragments and distances between them are much desirable since they give important information on the balance between the interparticle repulsion and their interaction with the random potential.

Another feasible experiment is the time-of-flight spectroscopy after switching off both the trap and the random potential. In this experiment the distribution of particles over momenta (velocities) is measured. The distribution of momentum has a finite width $\Delta p$. According to the uncertainty principle the value $\hbar / \Delta p$ can be treated as the average size of the fragment $R=\mathcal{L} / \ln \Gamma$ for the uncorrelated disorder [see the definition of $\Gamma$ in Eq. (76)]. The fastest is the dependence of this value on the strength of disorder: $\Delta p \propto U_{0}^{2}$. Others dependencies are logarithmic. For example, $\frac{d \Delta p}{d \ln \omega}=-\frac{3 \hbar}{\mathcal{L}}$ $=3 \frac{d \Delta p}{d \ln N}$. Installing a counter close to the trap at a distance comparable to the size of the trap would allow to register the oscillations of the particle flux due to discrete character of the fragmented state. This is another opportunity to find the distances between fragments. In the case of strongly correlated disorder the width of the distribution is determined by Eq. (66), in which the ratio $n_{c} / n$ must be substituted by $\Gamma$ defined by Eq. (77). In this case $\Delta p$ depends linearly on $\omega$. Other dependencies are much weaker. Nevertheless, the prediction that the ratio $\Delta p / \omega$ depends on one dimensionless parameter $\Gamma$ can be experimentally checked.

The transition between localized and delocalized coherent state in the random potential was confirmed in several experiments (see Sec. I). We propose to make more detailed measurement of the transition manifold and check our predictions formulated in Eqs. (27) and (65). 
An important question is whether the relaxation to the ground state can be reached during a reasonable time interval compatible with the time of experiment. We analyze this question for the uncorrelated or weakly correlated disorder. In this case the relaxation time due to tunneling can be estimated as $\tau=2 \pi \omega_{n}^{-1} t^{-1}$, where $\omega_{n} \sim \frac{\mathcal{E}}{\hbar}(\ln \Gamma)^{2}$ is the characteristic frequency of the optimal potential well and $t$ $\sim \exp \left[-\Gamma^{1 / 3}\right]$ is the tunneling coefficient [see Eq. (30)]. For numerical estimates we accept $\Gamma \sim 125, \ell \sim 10 \mu \mathrm{m}, b \sim \mathcal{L}$ $\sim 1 \mu \mathrm{m}, a \sim 0.01 \mu \mathrm{m}$, and $N \simeq 27000$. Then $t^{-1}=148$ and $\tau \sim 0.06 s$. The Larkin length can be increased by decreasing the amplitude of the random potential $\left(\mathcal{L} \propto U_{0}^{-2}\right)$. Simultaneously at fixed values $N, \ell$, and $a$ the value $\Gamma$ decreases as $\mathcal{L}^{-5}$. This example shows that the equilibrium is accessible, though it may be difficult to reach large ratio $\mathcal{L} / b$. In the experimentally important case of 1D (cigarlike) cloud the relaxation time is shorter since it is proportional to $\exp ($ $\left.-\Gamma_{1}\right)$ with $\Gamma_{1}=\ell^{2} / 3 N a \mathcal{L}$. At $\ell=25 \mu \mathrm{m}, \mathcal{L}=1 \mu \mathrm{m}$, and $a$ $=0.01 \mu \mathrm{m}$ the ratio $\Gamma_{1}$ reaches value 1 at $N \sim 85000$.

The closest to ours was the approach developed in the work by Lugan et al. ${ }^{22}$ Apart from the fact that these authors considered only the one-dimensional case, the main difference between our and their problems is that they considered the random potential with the exact lower boundary $U_{b}$ and with on-site distribution function $W[U] \propto \exp \left[-c\left(U-U_{b}\right)\right]$ instead of the Gaussian distribution. Such a distribution allows deeply localized states only at energies $E$ close to the exact lower boundary $U_{b}$. The corresponding fluctuations have the width $R$ the broader the closer $E$ is to $U_{b}$. It is clear that these levels are very different from those discussed above. If the random potential has the exact lower boundary, our theory is valid only if this boundary is separated from the most probable value of the potential by an energy interval strongly exceeding the energy dispersion. Then the localized states of our theory appear at intermediate energies between dispersion and $U_{b}$.

In conclusion, we presented a simple physical picture of deeply localized states of the Bose gas in a random potential. We demonstrated that the particles eventually fill the deep potential wells formed by fluctuations of the random potential and by their self-consistent field. Based on this idea the geometrical and physical properties of these states, such as the size of the clusters, the distances and the tunneling amplitudes between them, and the size of the whole cloud formed by the gas in a harmonic trap were calculated with precision of numerical coefficients. We discovered that the physical properties of these states depend significantly on correlations of the random potential. It occurs that the ground state of the system can be either almost homogeneous and coherent (superfluid) if the disorder is weak enough or fragmented and strongly localized. In particular, if the disorder is much stronger than the repulsion between particles, the system transits into a nonergodic state, whose properties even in the equilibrium strongly depend on the specific sample. At growing number of particles the system occurs in an ergodic but strongly localized ground state consisting of multiple particle clusters populating deep fluctuation wells. At the number of particles increasing, the tunneling between different potential wells increases leading to the phase correlation and finally to the quantum phase transition to the co- herent (superfluid) state. We have found a simple expression for the gas density at this transition in the gas confined by a big box and equation for the phase transition manifold in the gas confined by the harmonic trap. We have found that the repulsion suppresses the localization in one and two dimensions. In the 3D system it induces the transition to the superfluid state at the energy level at which single-particle states are still localized.

Quite recently there appeared a work by A. Babichenko and V. Babichenko ${ }^{72}$ in which the authors have formulated the problem of deeply localized state for weakly interacting Bose gas employing the Keldysh-Schwinger technique. For the uncorrelated Gaussian disorder they have found the instanton solution corresponding to the deeply localized state at a fixed chemical potential and the expression for the chemical potential vs given average density $n$, which coincides with our Eq. (29). Thus, they confirmed our theory by exact calculation.

\section{ACKNOWLEDGMENTS}

The authors acknowledge helpful support from the DFG through Grants No. NA222/5-2 and No. SFB 680/D4 (T.N.) as well as from the DOE under Grant No. DE-FG0206ER46278.

\section{APPENDIX: THE SUPERFLUID-INSULATOR TRANSITION IN ONE DIMENSION}

In this appendix we discuss the relation of our approach to the theory of Giamarchi and Schulz. ${ }^{33}$ It is convenient to state first the relation between the mass $m$, the boson density $n$, the scattering length $a$, and the parameters $K$ and $\kappa$ of the field theory of $1 \mathrm{D}$ bosons. ${ }^{33,73,74}$ We omit all subscripts 1 which are excessive for one dimension. The phonon velocity $v$ is then given by

$$
v=\left(\frac{n}{m \varkappa}\right)^{1 / 2},
$$

where $\varkappa$ denotes the compressibility of the bosons

$$
\varkappa=\frac{\partial n}{\partial \mu}=\frac{1}{\pi \hbar v K} .
$$

The Haldane inverse compressibility $\varkappa_{H}$ (Ref. 73) is related to our standard definition by $\varkappa=n^{2} / \varkappa_{H}$. Indeed, according to our definition the elastic energy per unit length is $E$ $\approx \frac{1}{2 \varkappa} \delta n^{2}$ which implies

$$
K=\frac{1}{\pi \hbar}\left(\frac{m}{n \varkappa}\right)^{1 / 2} .
$$

The Boson coupling constant $K$ is inverse to that of fermions. These relations agree with those of Ref. 31 taking into account that in Ref. 31 the density is dimensionless and $\hbar=1$. To get the relation between $a$ and $K$ we quote Haldane ${ }^{73}$ referring to earlier work of Lieb. ${ }^{75}$ Using the dimensionless parameter $\gamma=1 /\left(\pi^{2} n a\right)$ (we absorbed a factor $1 / \pi^{2}$ in the definition of $\gamma$ ), $K$ is related to $\gamma$ by 


$$
\begin{gathered}
K \approx \gamma^{1 / 2}\left(1-\frac{1}{2} \gamma^{1 / 2}\right), \quad \gamma \ll 1, \\
K \approx\left(1-\frac{8}{\pi^{2} \gamma}\right)^{1 / 2}, \quad \gamma \gg 1,
\end{gathered}
$$

with a smooth interpolation in between. Thus, $K$ changes continuously from 0 (for $a \rightarrow \infty$ corresponding to no interaction) to $K=1$ (for infinitely strong interaction). Since for $K$ $=1$ bosonic and fermionic descriptions coincide, an infinitely strong repulsive interaction corresponds to free fermions. ${ }^{76}$ Let us formulate the condition of superfluidity in terms of coupling constant $K$. The condition of superfluidity for $1 \mathrm{D}$ disordered system $n>n_{c} \approx a / \mathcal{L}^{2}$ [see Eq. (94)] can be rewritten as

$$
K>K_{\kappa}=\frac{1}{\pi n}\left(\frac{m \kappa}{\hbar^{2}}\right)^{2 / 3} \equiv \frac{1}{\pi n \mathcal{L}},
$$

where $\kappa$ denotes the disorder strength. We will show that $K \approx K_{K}$ remains the phase boundary between the superfluid and Bose glass phases also if quantum fluctuations are taken into account.

Let us study how quantum fluctuations renormalize the disorder strength $\kappa$. In lowest order in $\kappa$ the disorder strength is renormalized by the Debye-Waller factor:

$$
\kappa_{\mathrm{eff}} \approx \kappa e^{-2\left\langle\varphi^{2}\right\rangle}
$$

Here

$$
2\left\langle\varphi^{2}\right\rangle=\frac{2 \pi}{K} \int_{\mathcal{L}^{-1}}^{\xi^{-1}} \frac{d^{2} q}{(2 \pi)^{2}} \frac{1}{q^{2}} \approx \frac{1}{K} \ln (\mathcal{L} / \xi) \approx \frac{1}{K} \ln \left(\frac{K}{K_{\kappa}}\right),
$$

where we have used the relation for small $K$. Thus, for $K$ $<K_{\kappa}$ there are no fluctuation corrections and our condition (94) tells us that there is no superfluidity. In the opposite case

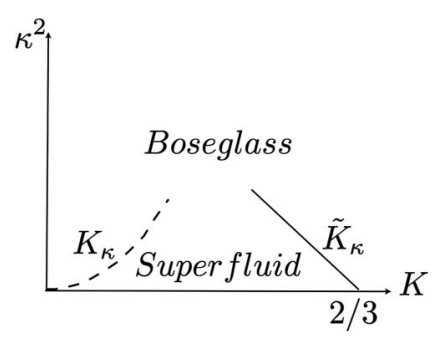

FIG. 8. Phase diagram in one dimension.

$K>K_{\kappa}$ we find (by making the calculation self-consistent) for $\max \left(K_{\kappa}, 2 / 3\right)<K$,

$$
\kappa_{\mathrm{eff}} \approx \kappa\left(\frac{K_{K}}{K}\right)^{1 /(K-2 / 3)} .
$$

Approaching the critical value $K=2 / 3$ the effective disorder strength is renormalized to zero for $K_{\kappa}<K<2 / 3$. In the opposite case there is no renormalization and the disorder is relevant. From these considerations it is clear that the transition happening at $K=2 / 3$ occurs only in one dimension. There is another transition line $K=K_{\kappa}$ which exists in higher dimensions. In the next order in $\kappa$, the condition $K=2 / 3$ changes to $K=\tilde{K}_{\kappa}$ with $\tilde{K}_{\kappa=0}=2 / 3 .{ }^{33}$ The transition at $K$ $=\widetilde{K}_{\kappa}$ is of the Kosterlitz-Thouless type. Even in a pure system superfluidity persists only when the healing length $\xi$ is smaller than the system size $L$, i.e., for $\xi \sim(a / n)^{1 / 2}<L$. For small $K$ this condition can be written as $\pi n K>1 / L$. Thus, for $a \rightarrow \infty$ the superfluidity persists only if

$$
K>K_{L}=\frac{1}{\pi n L} \text {. }
$$

The phase portrait obtained in this work is reproduced in Fig. 8. An extension at sufficiently strong disorder of the transition line found by Giamarchi and Schultz has been discussed by Altman et al. in Ref. 77 .
${ }^{1}$ P. W. Anderson, Phys. Rev. 109, 1492 (1958).

${ }^{2}$ E. Abrahams, P. W. Anderson, D. C. Licciardello, and T. V. Ramakrishnan, Phys. Rev. Lett. 42, 673 (1979).

${ }^{3}$ P. A. Lee and T. V. Ramakrishnan, Rev. Mod. Phys. 57, 287 (1985).

${ }^{4}$ I. M. Lifshitz, Zh. Eksp. Teor. Fiz. 53, 743 (1966).

${ }^{5}$ J. Zittartz and J. Langer, Phys. Rev. 148, 741 (1966).

${ }^{6}$ B. I. Halperin and M. Lax, Phys. Rev. 153, 802 (1967).

${ }^{7}$ B. Altshuler and A. Aronov, Pis'ma Zh. Eksp. Teor. Fiz. 27, 700 (1978).

${ }^{8}$ A. M. Finkelstein, Sov. Phys. JETP 57, 97 (1983); Sov. Sci. Rev. 14, 1 (1990).

${ }^{9}$ E. Abrahams, S. V. Kravchenko, and M. P. Sarachik, Rev. Mod. Phys. 73, 251 (2001).

${ }^{10}$ A. J. Leggett, Rev. Mod. Phys. 73, 307 (2001).

${ }^{11}$ J. R. Schrieffer and M. Tinkham, Rev. Mod. Phys. 71, S313 (1999).

${ }^{12}$ W. Ketterle, Rev. Mod. Phys. 74, 1131 (2002).
${ }^{13}$ F. D. Dalfovo, S. Giorgini, L. P. Pitaevskii, and S. Stringari, Rev. Mod. Phys. 71, 463 (1999).

${ }^{14}$ D. Snoke, Science 298, 1368 (2002).

${ }^{15}$ T. Giamarchi, C. Rüegg, and O. Tchernyshyov, Nat. Phys. 4, 198 (2008).

${ }^{16}$ V. F. Gantmakher, L. V. Golubkov, V. T. Dolgopolov, G. E. Tsydynzhapov, and A. A. Shashkin, JETP Lett. 68, 363 (1998).

${ }^{17}$ J. D. Reppy, J. Low Temp. Phys. 87, 205 (1992).

${ }^{18}$ C. L. Vicente, H. C. Choi, J. S. Xia, W. P. Halperin, N. Mulders, and Y. Lee, Phys. Rev. B 72, 094519 (2005).

${ }^{19}$ J. E. Lye, L. Fallani, M. Modugno, D. S. Wiersma, C. Fort, and M. Inguscio, Phys. Rev. Lett. 95, 070401 (2005).

${ }^{20}$ T. Schulte, S. Drenkelforth, J. Kruse, W. Ertmer, J. Arlt, K. Sacha, J. Zakrzewski, and M. Lewenstein, Phys. Rev. Lett. 95, 170411 (2005).

${ }^{21}$ L. Fallani, J. E. Lye, V. Guarrera, C. Fort, and M. Inguscio, Phys. Rev. Lett. 98, 130404 (2007).

${ }^{22}$ P. Lugan, D. Clement, P. Bouyer, A. Aspect, M. Lewenstein, and 
L. Sanchez-Palencia, Phys. Rev. Lett. 98, 170403 (2007).

${ }^{23}$ L. Sanchez-Palencia, D. Clement, P. Lugan, P. Bouyer, G. V. Shlyapnikov, and A. Aspect, Phys. Rev. Lett. 98, 210401 (2007); L. Sanchez-Palencia, D. Clement, P. Lugan, P. Bouyer, and A. Aspect, New J. Phys. 10, 045019 (2008).

${ }^{24}$ Yong P. Chen, J. Hitchcock, D. Dries, M. Junker, C. Welford, and R. G. Hulet, Phys. Rev. A 77, 033632 (2008).

${ }^{25}$ J. Billy, V. Josse, Z. Zuo, A. Bernard, B. Hambrecht, P. Lugan, D. Clement, L. Sanchez-Palencia, P. Bouyer, and A. Aspect, Nature (London) 453, 891 (2008).

${ }^{26}$ L. Fallani, C. Fort, and M. Inguscio, Adv. At., Mol., Opt. Phys. 56, 119 (2008).

${ }^{27}$ M. Rasolt, M. J. Stephen, M. E. Fisher, and P. B. Weichman, Phys. Rev. Lett. 53, 798 (1984).

${ }^{28}$ P. B. Weichman, M. Rasolt, M. E. Fisher, and M. J. Stephen, Phys. Rev. B 33, 4632 (1986).

${ }^{29}$ P. B. Weichman and M. E. Fisher, Phys. Rev. B 34, 7652 (1986).

${ }^{30}$ M. Ma, B. I. Halperin, and P. A. Lee, Phys. Rev. B 34, 3136 (1986).

${ }^{31}$ M. P. A. Fisher, P. B. Weichman, G. Grinstein, and D. S. Fisher, Phys. Rev. B 40, 546 (1989).

${ }^{32}$ P. B. Weichman and R. Mukhopadhyay, Phys. Rev. Lett. 98, 245701 (2007).

${ }^{33}$ T. Giamarchi and H. J. Schulz, Europhys. Lett. 3, 1287 (1987); Phys. Rev. B 37, 325 (1988).

${ }^{34}$ P. B. Weichman, Mod. Phys. Lett. B 22, 2623 (2008).

${ }^{35}$ D. K. K. Lee and J. M. F. Gunn, J. Phys.: Condens. Matter 2 , 7753 (1990); 4, 1729 (1992); J. Low Temp. Phys. 89, 101 (1992).

${ }^{36}$ K. Huang and H. F. Meng, Phys. Rev. Lett. 69, 644 (1992).

${ }^{37}$ S. Giorgini, L. Pitaevskii, and S. Stringari, Phys. Rev. B 49, 12938 (1994).

${ }^{38}$ A. V. Lopatin and V. M. Vinokur, Phys. Rev. Lett. 88, 235503 (2002).

${ }^{39}$ G. M. Falco, A. Pelster, and R. Graham, Phys. Rev. A 75, 063619 (2007); 76, 013624 (2007).

${ }^{40}$ R. Graham and A. Pelster, arXiv:cond-mat/0508306, Int. J. Bifurcation Chaos (to be published).

${ }^{41}$ V. I. Yukalov, E. P. Yukalova, K. V. Krutitsky, and R. Graham, Phys. Rev. A 76, 053623 (2007).

${ }^{42}$ A. Priyadarshee, S. Chandrasekharan, J. W. Lee, and H. U. Baranger, Phys. Rev. Lett. 97, 115703 (2006).

${ }^{43}$ F. Zhou, Phys. Rev. B 73, 035102 (2006)

${ }^{44}$ T. Paul, P. Schlagheck, P. Leboeuf, and N. Pavloff, Phys. Rev. Lett. 98, 210602 (2007).

${ }^{45}$ P. Lugan, D. Clement, P. Bouyer, A. Aspect, and L. SanchezPalencia, Phys. Rev. Lett. 99, 180402 (2007).

${ }^{46}$ S. E. Skipetrov, A. Minguzzi, B. A. van Tiggelen, and B. Shapiro, Phys. Rev. Lett. 100, 165301 (2008).

${ }^{47}$ T. Nattermann and V. L. Pokrovsky, Phys. Rev. Lett. 100, 060402 (2008).

${ }^{48}$ B. I. Shklovskii, Semiconductors 42, 909 (2008).

${ }^{49}$ L. V. Keldish and G. P. Proshko, Fiz. Tverd. Tela (Leningrad) 5, 3379 (1963).

${ }^{50}$ O. E. Kane, Phys. Rev. 131, 79 (1963).

${ }^{51}$ J. Cardy, J. Phys. C 11, L321 (1978).

${ }^{52}$ V. L. Bonch-Bruevich, Itogi Nauki-Serya "Fiz. Tverd. Tela"
(VINITI, Moscow, 1965).

${ }^{53}$ V. L. Bonch-Bruevich and A. G. Mironov, Vestn. Mosk. Univ., Ser. 3: Fiz., Astron. 6, 722 (1964).

${ }^{54}$ I. V. Andreev, Zh. Eksp. Teor. Fiz. 48, 1437 (1965).

${ }^{55}$ B. I. Shklovskii and A. L. Efros, Fiz. Tekh. Poluprovodn. 4, 305 (1970); Zh. Eksp. Teor. Fiz. 58, 657 (1970).

${ }^{56}$ S. John and M. J. Stephen, J. Phys. C 17, L559 (1984).

${ }^{57}$ D. Thirumalai, J. Phys. C 19, L397 (1986).

${ }^{58}$ B. I. Shklovsky and A. L. Efros, Electronic Properties of Doped Semiconductors (Springer, Berlin, 1984).

${ }^{59}$ G. M. Falco, T. Nattermann, and V. L. Pokrovsky, Europhys. Lett. 85, 30002 (2009).

${ }^{60}$ A. I. Larkin, Zh. Eksp. Teor. Fiz. 58, 1466 (1970).

${ }^{61}$ A. I. Larkin and Yu. N. Ovchinnikov, J. Low Temp. Phys. 34, 409 (1979).

${ }^{62}$ Y. Imry and S. K. Ma, Phys. Rev. Lett. 35, 1399 (1975).

${ }^{63}$ A. D. Mirlin and F. Evers, Phys. Rev. B 62, 7920 (2000).

${ }^{64}$ M. A. Skvortsov, V. E. Kravtsov, and M. V. Feigel'man, JETP Lett. 68, 84 (1998).

${ }^{65}$ The value which can be defined exactly is the density $n_{w}(E)$ of states with the energy less than $E: n_{w}(E)=\int_{-\infty}^{E} \nu(E) d E$. For energies corresponding to deeply localized states $(E<0$ and $|E| \gg \mathcal{E})$ this value coincides with $n_{w}(R)$ at $R=\hbar / \sqrt{2 m|E|}$.

${ }^{66}$ L. D. Landau and E. M. Lifshitz, Quantum Mechanics, 3rd ed. (Pergamon, New York, 1991).

${ }^{67}$ N. N. Bogoliubov, J. Phys. (USSR) 11, 23 (1947).

${ }^{68}$ S. T. Belyaev, Sov. Phys. JETP 7, 104 (1958); 7, 289 (1959).

${ }^{69}$ The universal equation (33) has been considered also in I. M. Lifshits, S. A. Gredeskul, and L. A. Pastur, Introduction to the Theory of Disordered System (Wiley, New York, 1988), pp. 229 230. There, the lowest eigenvalue is calculated imposing the boundary condition $\phi(0)=1$. Differently, we have fixed the normalization of the solution, namely, $\int \phi^{2} d^{3} x=1$.

${ }^{70}$ L. P. Pitaevskii and S. Stringari, Bose-Einstein Condensation (Clarendon Press, Oxford, 2003).

${ }^{71}$ The regime diagram is the analog of the phase diagram. The difference is that the sharp phase transitions are realized only in infinite systems, whereas in finite systems more or less smooth crossovers between different regimes take place.

${ }^{72}$ A. Babichenko and V. Babichenko, Phys. Lett. A 373, 2973 (2009).

${ }^{73}$ F. D. M. Haldane, Phys. Rev. Lett. 47, 1840 (1981).

${ }^{74}$ Quasi-one-dimensional system in experiments with cooled gases was realized as a narrow cylinder (or ellipsoid) of the length $\ell$ and transverse extension $\ell_{\perp}$, with $\ell \gg \ell_{\perp} \gg a$. In this case we get $a_{1} \sim \ell_{\perp}^{2} / a$ and $n_{1}=n \ell_{\perp}^{2}$, respectively. The dimensionless interaction parameter can be rewritten as $\left(a_{1} n_{1}\right)^{-1}=a n^{1 / 3}\left(n^{1 / 3} \ell_{\perp}\right)^{-4}$. Thus, if the gas parameter $a n^{1 / 3}$ is small and the radius of the cylinder is larger than the mean particle distance, we get $\left(a_{1} n_{1}\right)^{-1} \ll 1$. In this region the coupling constant $K$ satisfies a following relation $K \sim \sqrt{a n^{1 / 3}}\left(n^{1 / 3} \ell_{\perp}\right)^{-2} \ll 1$.

${ }^{75}$ E. H. Lieb, Phys. Rev. 130, 1616 (1963).

${ }^{76}$ A. De Martino, M. Thorwart, R. Egger, and R. Graham, Phys. Rev. Lett. 94, 060402 (2005).

${ }^{77}$ E. Altman, Y. Kafri, A. Polkovnikov, and G. Refael, Phys. Rev. Lett. 93, 150402 (2004); 100, 170402 (2008); arXiv:0909.4096 (unpublished). 